\title{
An overview of the main foodstuff sample preparation technologies for tetracycline residue determination
}

\author{
Michael Pérez-Rodríguez ${ }^{\mathrm{a}, \mathrm{b}, *}$, Roberto Gerardo Pellerano ${ }^{\mathrm{a}}$, Leonardo Pezza ${ }^{\mathrm{b}}$, \\ Helena Redigolo Pezza ${ }^{\mathrm{b}}$ \\ ${ }^{a}$ Institute of Basic and Applied Chemistry of the Northeast of Argentina (IQUIBA-NEA), National Scientific and Technical Research Council (CONICET), Faculty of \\ Natural and Exact Sciences and Surveying, National University of the Northeast - UNNE, Av. Libertad 5470, Corrientes 3400, Argentina \\ ${ }^{\mathrm{b}}$ Institute of Chemistry, São Paulo State University - UNESP, R. Prof. Francisco Degni 55, P.O. Box 355, 14800-900 Araraquara, SP, Brazil
}

\section{A R T I C L E I N F O}

\section{Keywords:}

Tetracyclines

Foodstuffs

Sample preparation

Extraction and clean-up

Residue determination

Instrumental analysis

\begin{abstract}
A B S T R A C T
Tetracyclines are widely used for both the treatment and prevention of diseases in animals as well as for the promotion of rapid animal growth and weight gain. This practice may result in trace amounts of these drugs in products of animal origin, such as milk and eggs, posing serious risks to human health. The presence of tetracycline residues in foods can lead to the transmission of antibiotic-resistant pathogenic bacteria through the food chain. In order to ensure food safety and avoid exposure to these substances, national and international regulatory agencies have established tolerance levels for authorized veterinary drugs, including tetracycline antimicrobials. In view of that, numerous sensitive and specific methods have been developed for the quantification of these compounds in different food matrices. One will note, however, that the determination of trace residues in foods such as milk and eggs often requires extensive sample extraction and preparation prior to conducting instrumental analysis. Sample pretreatment is usually the most complicated step in the analytical process and covers both cleaning and preconcentration. Optimal sample preparation can reduce analysis time and sources of error, enhance sensitivity, apart from enabling unequivocal identification, confirmation and quantification of target analytes. The development and implementation of more environmentally friendly analytical procedures, which involve the use of less hazardous solvents and smaller sample sizes compared to traditional methods, is a rapidly increasing trend in analytical chemistry. This review seeks to provide an updated overview of the main trends in sample preparation for the determination of tetracycline residues in foodstuffs. The applicability of several extraction and clean-up techniques employed in the analysis of foodstuffs, especially milk and egg samples, is also thoroughly discussed.
\end{abstract}

\section{Introduction}

Tetracyclines (TCs) are a natural or semisynthetic group of antibiotics that exhibits antimicrobial activity against a wide range of gram- positive and gram-negative bacteria $[1,2]$. These antibiotics have been widely employed in human medicine for the treatment of infectious diseases [3]. The substances possess very similar chemical structures derived from a common core hydronaphthacene containing four fused

\footnotetext{
Abbreviations: TCs, tetracyclines; TC, tetracycline; OTC, oxytetracycline; CTC, chlortetracycline; DC, doxycycline; MRL, maximum residue limit; HPLC, high performance liquid

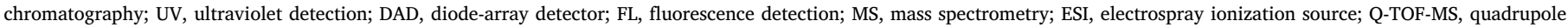

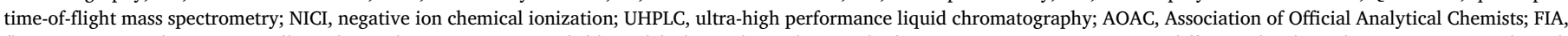

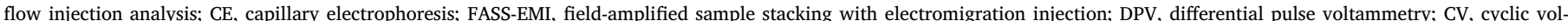

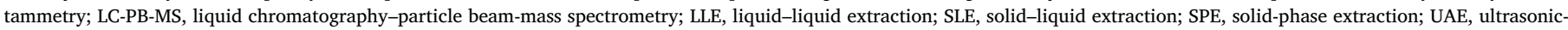

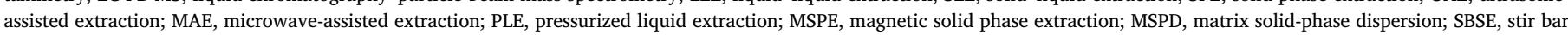

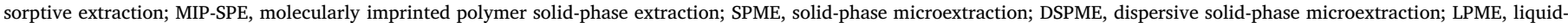

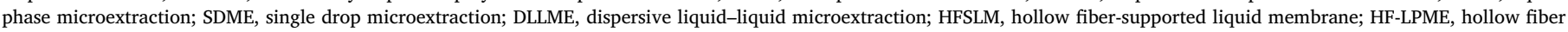

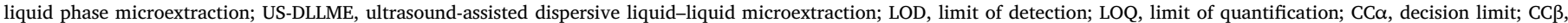

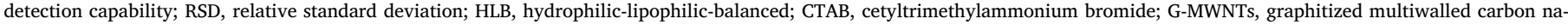

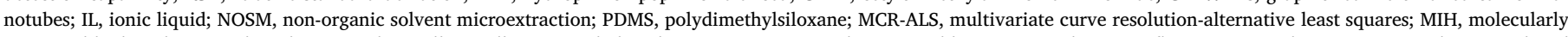

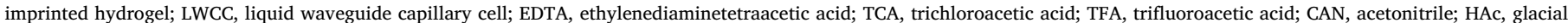
acetic acid; $\mathrm{MeOH}$, methanol; ZIF-8, zeolite imidazolate framework-8

* Corresponding author at: Institute of Basic and Applied Chemistry of the Northeast of Argentina (IQUIBA-NEA), National Scientific and Technical Research Council (CONICET), Faculty of Natural and Exact Sciences and Surveying, National University of the Northeast - UNNE, Av. Libertad 5470, Corrientes 3400, Argentina.

E-mail addresses: michaelpr1984@gmail.com, michaelpr@iq.unesp.br (M. Pérez-Rodríguez).
} 


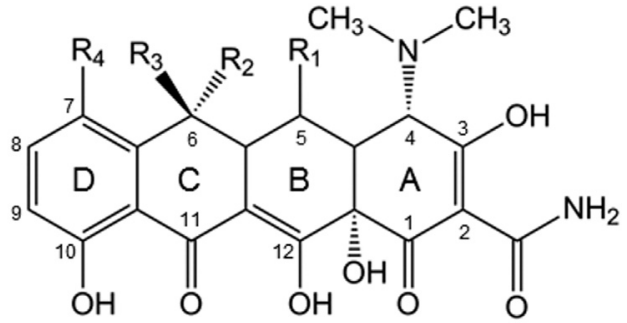

\begin{tabular}{lllll}
\hline Compound & $\mathrm{R}_{1}$ & $\mathrm{R}_{2}$ & $\mathrm{R}_{3}$ & $\mathrm{R}_{4}$ \\
\hline Tetracycline & $\mathrm{H}$ & $\mathrm{CH}_{3}$ & $\mathrm{OH}$ & $\mathrm{H}$ \\
Oxytetracycline & $\mathrm{OH}$ & $\mathrm{CH}_{3}$ & $\mathrm{OH}$ & $\mathrm{H}$ \\
Chlortetracycline & $\mathrm{H}$ & $\mathrm{CH}_{3}$ & $\mathrm{OH}$ & $\mathrm{Cl}$ \\
Doxycycline & $\mathrm{OH}$ & $\mathrm{CH}_{3}$ & $\mathrm{H}$ & $\mathrm{H}$ \\
\hline
\end{tabular}

Fig. 1. Chemical structure of the most commonly used tetracyclines.

rings. The common chemical structure of tetracyclines has three functional groups that influence the permeability of the molecule across biological membranes, thereby leading to the absorption of the tetracycline molecule by bacterial cells [4].

Tetracyclines are strong chelating agents, with the primary site of chelation being the 11, 12 B diketone system (Fig. 1). Chelation of a divalent metal ion to tetracyclines is essential for the antimicrobial activity of the antibiotic; such coordination is regarded very promising in the search for new compounds capable of reversing bacterial resistance $[4,5]$. The coordination of platinum with tetracycline and doxycycline via A ring yields compounds that are active against bacterial strains resistant to tetracycline and other antibiotics. By virtue of that, coordination compounds containing tetracyclines may be used in the future in clinical medicine for the treatment of cancer and other infectious diseases [5].

Due to their broad spectrum activity and low cost, tetracyclines are widely administered in food-producing animal husbandry for preventive and curative purposes $[2,6]$. These drugs are also employed as additives in animal feed aiming at promoting rapid animal growth and weight gain $[1,7,8]$. The rampant application of the drugs is currently regarded a serious problem for public health systems worldwide; this is largely because the widespread use of these substances induces an increase in infections caused by strains resistant to antimicrobial agents. In this sense, conditions hazardous to human and animal health are attributed to such practice, since most conventional treatments are rendered ineffective for antimicrobial clinical care $[7,8]$. Currently, there are eight marketed tetracyclines, with four of them being most commonly used in the veterinary prescription of antimicrobials [9]: tetracycline (TC), oxytetracycline (OTC), chlortetracycline (CTC) and doxycycline (DC) (Table 1).

Over the years, the indiscriminate use of tetracycline drugs in animal feed has raised huge concerns regarding the quality of food, including honey, meat, fish, milk and eggs, available to consumers $[4,10,11]$. The contamination of these types of foods with tetracycline residues poses risk to human health, having the potential of causing harmful effects on the population. Such deleterious consequences may range from possible allergic reactions, liver damage, teeth yellowing to gastrointestinal disturbances, among others. Another negative effect is that the consumption of foods contaminated with tetracycline residues is likely to induce the increase of pathogens resistant to antimicrobial agents [2].

The relationship between the use of antimicrobials in animal husbandry and antimicrobial resistance in humans has become a matter of great concern to public health. The reason being that the indiscriminate treatment of animals with veterinary antibiotics can turn their derived food products into sources for resistance to antibiotics in human species
$[8,12]$. The discovery of new resistant strains of bacteria and other microorganisms that are becoming increasingly resistant over time has led to an increasing pressure on laboratories responsible for food safety. The laboratories are expected to monitor the use of veterinary pharmaceuticals and ensure food quality for human consumption [2,4].

\subsection{Regulatory authorities}

An important area in the food sector is the management of food and nutrition safety. The role of the professionals in this area is to ensure access to quality basic food, in sufficient quantity, permanently and without compromising access to other essential needs based on healthy eating practices. Clearly, the relevance of the role played by the professionals in this area needs not be overemphasized. Their activities contribute to a dignified existence, in a comprehensive development context, of human beings $[13,14]$. Essentially, foodstuffs are required to be free from any form of contamination in order to ensure human safety.

To protect human health from the adverse effects of potentially dangerous antibiotics, several regulatory authorities have established tolerance levels or maximum residue limits (MRLs) for substances used as veterinary drugs in food-producing animals. MRLs have been defined by the European Community as the maximum concentration of residue of a veterinary product present in foodstuffs of animal origin, which may be ingested daily without posing any toxicological hazard to human health. In other words, MRLs are levels of a drug accepted by the community to be legally permitted or recognized as acceptable in food, and are expressed in $\mathrm{mg} \mathrm{kg}^{-1}$ or $\mu \mathrm{gg}^{-1}[11,12]$.

The Codex Alimentarius is a worldwide reference for consumers, food producers and processors, national food control agencies and other stakeholders in international food trade. The Codex Alimentarius food standards are internationally acknowledged as the best-established measures suitable for the protection of consumer health in addition to promoting fair practices in the food industry. Consumers can rely on the safety and quality of the food products they acquire while importers can rest assured that the food they order will meet their specifications $[15,16]$.

Table 2 lists the MRLs established by Codex Alimentarius [17], European Union (EU) [18], Canada [19], People's Republic of China [20] and Brazil [21] with specific regulations for tetracycline (TC), oxytetracycline (OTC), and chlortetracycline (CTC) in some animalderived food. In Brazil, tetracyclines are legally regulated in accordance with the National Plan for the Control of Residues and Contaminants (Plano Nacional de Controle de Resíduos e Contaminantes, PNCRC) [21]. These tolerance limits have been adopted for both individual tetracyclines and their combinations. For instance, in the case of bovine milk, the Brazilian regulation (PNCRC) [21] also includes doxycycline (DC) within the combination of these residues. When it comes to eggs, the European Union [18] and China [20] have relatively stricter regulations, with a tolerance limit of $200 \mu \mathrm{g} \mathrm{kg}^{-1}$ for this class of compounds. Milk and eggs exhibit lower MRLs, as such, their quality control is, undoubtedly, of extreme importance.

The presence of tetracycline residues in animal-derived foods at levels above the legally tolerable limit indicates failure of compliance with good veterinary practices. This lack of compliance with established regulations renders the products unqualified and improper for human consumption. For these reasons, there is an unquestionable need for the stakeholders involved to set out effective and adequate means of monitoring and determining traces of these antibiotics in the most varied foodstuffs to help meet the requirements of regulatory agencies. Indeed, effective control of these residues is essential if we are to ensure a high degree of consumer protection.

Relevant data were obtained by searching through SciFinder and Scopus regarding the main methods for the analysis of honey, milk, eggs and animal tissues containing the term "tetracycline" in the title, abstract or keywords. The data indicate that since 1996 there has been 
Table 1

Some chemical and physical properties of tetracycline antimicrobials (adapted from $[4,57,138]$ ).

\begin{tabular}{|c|c|c|c|c|c|}
\hline Antimicrobial & Systematic name & Chemical structure & $\begin{array}{l}\text { Acidity } \\
\text { (pka) }\end{array}$ & $\begin{array}{l}\text { Polarity } \\
(\log P)\end{array}$ & $\begin{array}{l}\text { Molecular } \\
\text { mass }(\mathrm{g} / \mathrm{mol})\end{array}$ \\
\hline Tetracycline (TC) & $\begin{array}{l}\text { 4-(Dimethylamino)-1,4,4a,5,5a,6,11,12a-octahydro-3,6,10,12,12a- } \\
\text { pentahydroxy-6-methyl-1,11-dioxo-2-naphthacene carboxamide }\end{array}$ & & $\begin{array}{l}\mathrm{pK}_{\mathrm{a} 1} 3.3 \\
\mathrm{pK}_{\mathrm{a} 2} 7.7 \\
\mathrm{pK}_{\mathrm{a} 3} 9.7\end{array}$ & -1.3 & 444.4 \\
\hline Oxytetracycline (OTC) & 5-hydroxy-tetracycline & & $\begin{array}{l}\mathrm{pK}_{\mathrm{a} 1} 3.2 \\
\mathrm{pK}_{\mathrm{a} 2} 7.5 \\
\mathrm{pK}_{\mathrm{a} 3} 8.9\end{array}$ & -3.6 & 460.4 \\
\hline Chlortetracycline (CTC) & 7-chloro-tetracycline & & 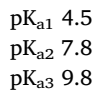 & -0.62 & 478.8 \\
\hline Doxycycline (DC) & 6-deoxy-5-hydroxy-tetracycline & & 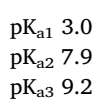 & -1.9 & 444.4 \\
\hline
\end{tabular}

Table 2

Maximum residue limits for tetracyclines in foods of animal origin.

\begin{tabular}{|c|c|c|c|c|}
\hline Authority & Animal group & Target food & $\begin{array}{l}\text { MRL } \\
\left(\mu \mathrm{kg}^{-1}\right)\end{array}$ & Ref. \\
\hline \multirow[t]{7}{*}{ Codex Alimentarius } & Poultry & Eggs & 400 & [17] \\
\hline & Poultry & Muscle & 200 & \\
\hline & Poultry & Liver & 600 & \\
\hline & Cattle & Milk & 100 & \\
\hline & Cattle and Swine & Muscle & 200 & \\
\hline & Cattle and Swine & Liver & 600 & \\
\hline & Fish & Muscle & 200 & \\
\hline \multirow[t]{6}{*}{ Canadian } & Poultry & Eggs & 400 & [19] \\
\hline & Poultry & Muscle & 200 & \\
\hline & Poultry & Liver & 600 & \\
\hline & Cattle & Milk & 100 & \\
\hline & Cattle and Swine & Muscle & 200 & \\
\hline & Cattle and Swine & Liver & 600 & \\
\hline \multirow[t]{4}{*}{ European Union } & Poultry & Eggs & 200 & [18] \\
\hline & $\begin{array}{l}\text { Poultry, Cattle and } \\
\text { Swine }\end{array}$ & Muscle & 100 & \\
\hline & $\begin{array}{l}\text { Poultry, Cattle and } \\
\text { Swine }\end{array}$ & Liver & 300 & \\
\hline & Cattle & Milk & 100 & \\
\hline \multirow[t]{4}{*}{ Chinese } & Poultry & Eggs & 200 & [20] \\
\hline & All Animal Source Foods & Muscle & 100 & \\
\hline & All Animal Source Foods & Liver & 300 & \\
\hline & Cattle and Lamb & Milk & 100 & \\
\hline \multirow[t]{4}{*}{ Brazilian (PNCRC) } & Poultry & Eggs & - & [21] \\
\hline & $\begin{array}{l}\text { Poultry, Cattle and } \\
\text { Swine }\end{array}$ & Muscle & 200 & \\
\hline & $\begin{array}{l}\text { Poultry, Cattle and } \\
\text { Swine }\end{array}$ & Liver & - & \\
\hline & Cattle & Milk & 100 & \\
\hline
\end{tabular}

a steady increase in the number of scientific publications related to the determination of these veterinary drugs in foodstuffs. The review was conducted based on 96 articles published between 1992 and 2017. There has been an increasingly growing interest among scientists in studying tetracyclines, regarded an important research subject in food safety issues.

Fig. 2 shows the distribution of the selected publications according to the analyzed foodstuffs. As can be seen, milk (35\%) and eggs (18\%) are the most frequently studied food matrices; this is probably due to

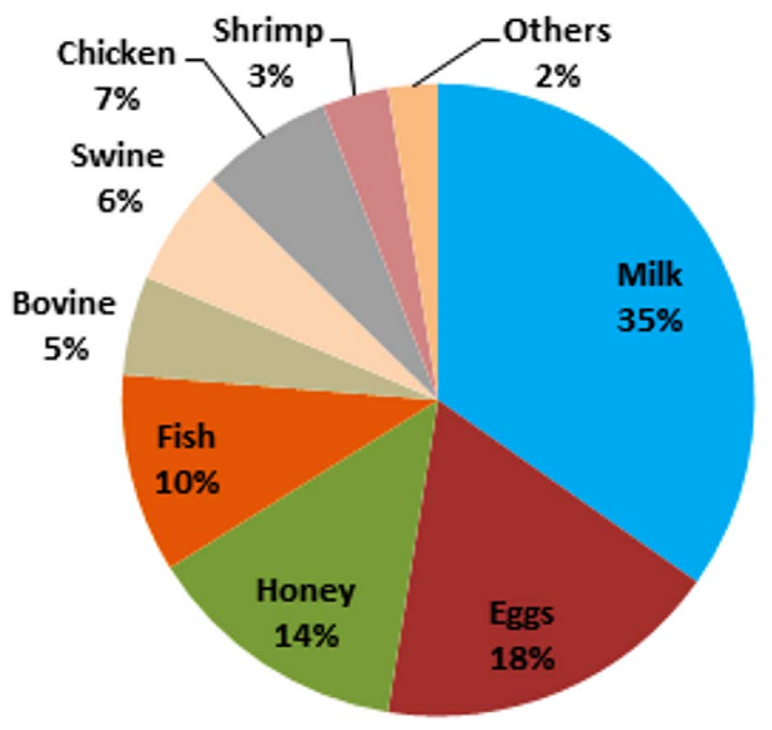

Fig. 2. Different types of animal-origin foods studied which are used for detecting traces of tetracyclines.

their low cost and the fact that they are easily accessible to consumers. Milk and eggs are animal-origin foods that deserve careful attention. This is largely because their high nutritional value provides health benefits and are therefore highly consumed by humans. They are important sources of proteins, vitamins and minerals [22,23] and constitute fundamental components of the diet consumed in Brazil and other countries. The habitual human consumption of milk and eggs is important for maintaining a balanced and healthy diet. Hence, the presence of any contaminant in these foods is strictly not allowed $[13,24]$.

The present review aims to investigate the main sample preparation techniques related to the analysis of tetracyline drugs in animal-derived foods, focusing on milk and egg matrices. This research will help identify aspects of residue extraction and sample clean-up that still require clarification. We will provide an overview of the parameters to consider for the development and implementation of a new food sample preparation method. To this end, 96 papers published between 1992 and 2017 have been thoroughly reviewed and are described below. 


\section{Analytical techniques}

A wide range of analytical methods have been developed for the analysis of tetracyclines in food matrices. Among the techniques that merit mentioning include high performance liquid chromatography (HPLC) with ultraviolet (UV), fluorescence (FL), or mass spectrometry (MS) detection. In fact, the AOAC International recommends the use of HPLC with UV detection as the official method for the analysis of tetracycline residues in foods of animal origin, such as cow milk [25] and swine and bovine tissues [26].

Tetracyclines are highly polar, water soluble compounds that undergo chelation with metal ions [27]. They are weak bases with pKa values ranging from 3.2 to 9.8 (Table 1) and contain several chromophore groups. In view of that, tetracyclines exhibit an intensive absorption band in the ultraviolet region between 270 and $360 \mathrm{~nm}$, in both acidic and neutral medium. UV and diode array (DAD) detectors are commonly used for the determination of this class of substances [5].

The mechanism by which tetracyclines are retained in chromatographic systems can be quite complex, in that these antibiotics present different functional groups. Reverse phase columns (C8 and C18) are commonly used for the chromatographic separation of these substances. The packing material allows tetracycline retention through the following mechanisms: ion pair formation, competition effects, active and inactive binding sites, ion exchange, interaction with silanol groups, among others $[28,29]$.

Because of the high polarity nature of tetracyclines, mobile phases with high water content are widely applied in the performance of the chromatographic analyses [28]. Moreover, these substances can be adsorbed by the silanol groups through the reverse phase column, and tend to exhibit peaks with tail and low resolution. These problems can be overcome by adding oxalic acid in the mobile phase and using polystyrene-divinylbenzene columns. This explains the reason why mobile phase containing oxalic acid has been frequently reported in the literature on the analysis of tetracyclines in foods using HPLC technique since the late 80 s. The mobile phase composition plays a very important role when it comes to the chromatographic behavior of tetracyclines [30].

Apart from HPLC, other techniques have also been employed toward the determination of tetracycline residues in food products. Fig. 3 shows the distribution of publications according to the analytical techniques applied. As mentioned above, among the target matrices, HPLC (69\%) was found to be the most commonly used technique for the analysis of tetracyclines. This is probably because its varied detection systems provide high sensitivity and selectivity. Other techniques that have also been employed include spectrophotometry (9\%), voltammetry (7\%), capillary electrophoresis (6\%), enzyme-linked

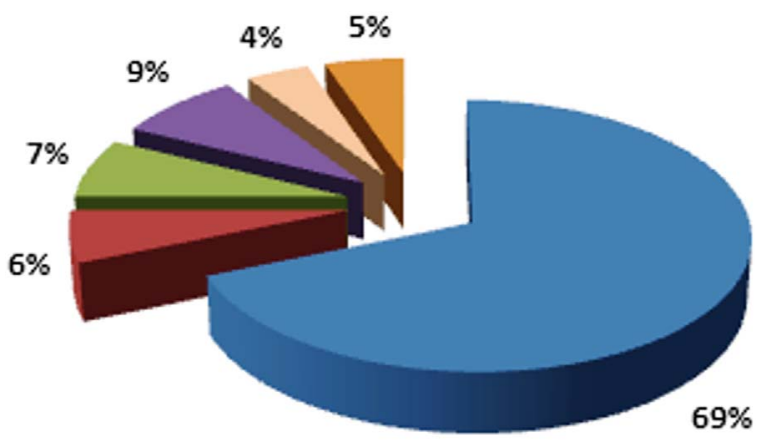

\section{DPLC \\ Voltammetry \\ Capillary electrophoresis \\ Luminescence \\ - Spectrophotometry \\ EUSA and others}

Fig. 3. Types of analytical techniques used for tetracycline determination in foodstuffs. immunosorbent assay (ELISA) (5\%), and luminescence (4\%). A single work reported in the literature used Bragg diffraction red-shift for the determination of this group of substances. There have also been reports of studies that blended spectrophotometry and luminescence techniques with flow injection analysis.

Despite advances in the sensitivity of modern analytical instrumentation for the determination of trace contaminants in complex matrices, such as food, a rigorous sample pre-treatment is often required for extracting and isolating the target analytes from the food matrix, and thus, facilitate their determination [31].

A wide range of methods for the determination of tetracycline residues in food products have been reported in the literature. Most of these methods employed in the determination obtained low limits of detection and good extraction capacities because of the use of adequate sample preparation prior to instrumental analyses. However, a greater part of the methods lacks sufficient sensitivity needed for detecting residues in accordance with the maximum limits allowed; this makes them time-consuming besides involving laborious preconcentration procedures. Such procedures are often expensive and generally employ considerable volumes of organic solvents, resulting in the generation of large quantities of toxic waste that hinders proper treatment of solvents, while endangering human health and contributing to environmental pollution.

The choice of sample treatment depends largely on the complexity of the matrix as well as on the selected detection method. Highly sensitive detection methods require careful sample treatment steps; this is because, most often, sensitivity increases in both the target analytes and coextractives, generating unwanted matrix interferences in the analyses [31].

A powerful thrust for determining various chemical components with minimal or even no sample preparation was reached by direct analysis in real time (DART) mass spectrometry (MS) - an ambient ionization technique. This ionization approach is based on the fundamental principles of atmospheric pressure chemical ionization (APCI). Here, metastable species are generated from an excited helium gas stream. The species subsequently react with analytes to form gaseous ions [32-35]. The main parameters that are known to affect the ionization efficiency of DART include desorption temperature, gas type, gas flow rate, discharge energy and angular disposal of the sampler, among others $[33,35]$.

The introduction of DART-MS accompanied by the rapid evolution of MS analyzers have led to the development of a simple and highthroughput frontier technology for rapid mass spectral analysis in situ of a large variety of samples. DART-MS allows one to analyze samples under atmospheric pressure. Furthermore, compounds that have been deposited or adsorbed onto surfaces or that are being desorbed thereof into the atmosphere can also be successfully analyzed with the aid of this technique $[33,34]$.

Over the last decade, the application field of the DART-MS technique has seen extraordinary expansion. The technique has gained popularity among researchers and has been considered suitable for the following: olive oil authenticity assessment [36], pesticide monitoring on fruits [37] and vegetables [38], metabolite fingerprinting [39], plant seed characterization [40], clinical [35], pharmaceutical [41], forensic [42] and environmental [43] analyses, and for the detection of adulteration in foods such as milk [44] and honey [45].

In comparison with the traditional chromatography coupled to electrospray mass spectrometry, DART-MS presents improvements in terms of analysis time (in seconds) and operational simplicity [43]. Nonetheless, since there is no separation step prior to ionization in DART, these types of procedures must be carefully designed in order to avoid signal suppression, which when very pronounced may produce lower detection selectivity. This shortcoming (lack of separation step) renders the DART-MS technique more vulnerable to false (negative or positive) results [32]. In this context, both the efficiency of the sample treatment and the mass resolution are of extreme relevance for analysis 
by the DART-MS technique [43].

Modern analytical strategies tend towards automatization and integration of sample preparation in the detection systems. These strategies are, most often, merged with chromatographic systems. Both solvent-minimized and solventless sample pretreatment techniques developed during the last decade are among the pillars of green analytical chemistry. The interest in this green approach is greatly driven by toxicological, environmental and economical concerns [31].

\section{Sample preparation}

Sample preparation is usually the most critical step in the development of methods for residue analysis. The type of treatment adopted depends on the matrix type, the method, and the chemical attributes of the analytes $[10,46]$. The typical steps involved in sample preparation include sampling, extraction, clean-up and concentration, followed by the final analysis. The sampling step is regarded the most important among them because an inadequate sampling can lead to erroneous results, regardless of whether the remaining steps in the analytical process have been flawless. Any portion or aliquot taken for analysis must be representative of the original bulk sample. For solid foodstuffs, proper crushing is recommendable to obtain smaller sample sizes. When conducting food composition analysis, smaller sample portions can be used. By contrast, for the determination of trace contaminants in food, relatively larger portions of sample are often required in order to obtain lower limits of detection. Irrespective of the selected sampling mechanism, sample homogenization is essential for chemical analysis [47].

Evidently, a large sample size requires more efficient extraction and clean-up techniques than a smaller one; hence, the economic costs of analysis are likely to increase [47]. Extraction methods are usually applied toward the isolation of residue contaminants from a representative sample, whereas clean-up methods are employed with the aim of separating co-extracted matrix components in order to obtain a more purified sample extract [31]. In analytical chemistry, these terms - extraction and clean-up - are commonly used interchangeably, and are often interdependent.

The extraction and clean-up methods for tetracycline residue analysis reported in the literature have been applied to a broad variety of matrices. Foodstuffs, such as milk and eggs, contain many interfering substances that need to be removed selectively. The difficulty encountered in the analysis of tetracycline antibiotics in this type of foods lies in the need to eliminate the main matrix components (e.g., lipids, proteins, vitamins, minerals, fats, and other nutritional and energy sources) that may interfere in the instrumental determination of the investigated compounds. This difficulty has led to the development of new strategies for the isolation and extraction of tetracycline residues from food matrices [10].

Table 3 summarizes the most relevant analytical applications regarding the determination of tetracycline drugs in different foodstuffs. The main techniques employed for the extraction and clean-up of tetracyclines from such matrices include solvent extraction (SE), solidphase extraction (SPE), ultrasonic-assisted extraction (UAE), pressurized liquid extraction (PLE), magnetic solid-phase extraction (MSPE), matrix solid-phase dispersion (MSPD), molecularly imprinted polymer solid-phase extraction (MIP-SPE), and dispersive liquid-liquid microextraction (DLLME). Other less common techniques include hollow fiber liquid phase microextraction (HF-LPME) and dispersive solidphase microextraction (DSPME). The advantages and limitations of these techniques are presented in Table 4.

The investigation conducted here showed that there are few methods capable of detecting traces of tetracyclines in animal-origin foods that do not include a prior sample treatment. This is probably due to the complexity of these matrices. Some authors have developed electrochemical methods using differential pulse voltammetry (DPV) [48] and cyclic voltammetry (CV) [49] for the analysis of milk matrix without further sample treatment. Another work published in the literature involving the use of honey and milk samples reported to have obtained good linearity within the matrix application ranges. The authors achieved this result by diluting milk and honey samples in water and subsequently measuring the content through Bragg diffraction redshift [50]. Although these methods showed good results, some sample preparation is normally required prior to analysis so as to avoid matrix interference [12]. In fact, a report in the literature indicates that very low concentrations of these antibiotics in some food matrices were determined without performing sample clean-up, or with only a single prior step of protein precipitation [46].

\subsection{Solvent extraction}

Due to easy binding of the tetracyclines with proteins, the addition of strong acids and precipitating agents is highly recommended when it comes to extracting these compounds from food samples [28]. Deproteinization is a simple solvent extraction (SE) procedure commonly used to extract antibiotics from complex matrices. This off-line procedure is often necessary for the removal of matrix interferences in order to ensure good recoveries of analytes of interest [12].

Protein precipitation in milk and egg samples involves the use of organic solvents including acetonitrile [6,10,23,46,51], methanol [52-55] or acetone [56], as well as the combination of these solvents with acid compounds, such as trichloroacetic acid [46,57-61], trifluoroacetic acid [62,63], glacial acetic acid [64-66], succinic acid $[67,68]$, citric acid $[23,69]$, formic acid $[54,70]$, or perchloric acid [71]. Other authors have used McIlvaine-EDTA [6,72-79], sodium citrate [10], oxalate [63], sodium succinate [80] and phosphate [81] buffers as deproteinizing agents for the treatment of these samples. Such processes are generally followed by some sample clean-up procedures.

Deproteinization of milk samples with $1 \%$ trichloroacetic acid solution without further clean-up procedure was reported to be sufficient for detecting tetracycline residues in remaining solution by spectrophotometry [46]. The acid treatment evaluated in that work yielded adequate recoveries (71.0-82.0\%), considering the complexity of the milk matrix. Moreover, the authors obtained relatively lower limits of detection compared to other studies that employed complicated sample treatments $[3,53]$ and expensive equipments $[67,71]$.

Liquid-liquid extraction (LLE) is a separation process based on the different distribution (relative solubility) of the analytes in two immiscible liquid phases (usually water and an organic solvent); the process is controlled by partition equilibrium. The extraction of an analyte depends on the transfer of its mass from a first liquid phase to a second one; this is achieved by the differences in solubilities between the phases [47]. LLE is one of the most commonly used extraction techniques for the extraction of many organic compounds from a large variety of matrices, particularly aqueous samples.

Tetracycline antibiotics in milk samples have been determined by capillary electrophoresis using a clean-up/extraction procedure based on protein precipitation with trichloroacetic acid followed by liquid-liquid extraction with dichloromethane [58]. The method showed adequate sensitivity for the detection of residues and the recoveries obtained were within the allowed limits.

Solid-liquid extraction (SLE), also known as leaching process, is another technique employed in solvent extraction which has similar principles to those of LLE. The only difference is that in SLE analytes are extracted from solid samples. The SLE process occurs through the displacement of soluble substances, and analytes are extracted using a solvent. Owing to the interaction between the phases, the analytes can diffuse from the solid sample to the liquid phase, leading to the separation of the main components.

In general, the techniques described above involve the use of large quantities of organic solvents, which contribute significantly toward environmental pollution. Apart from their negative impact on the 


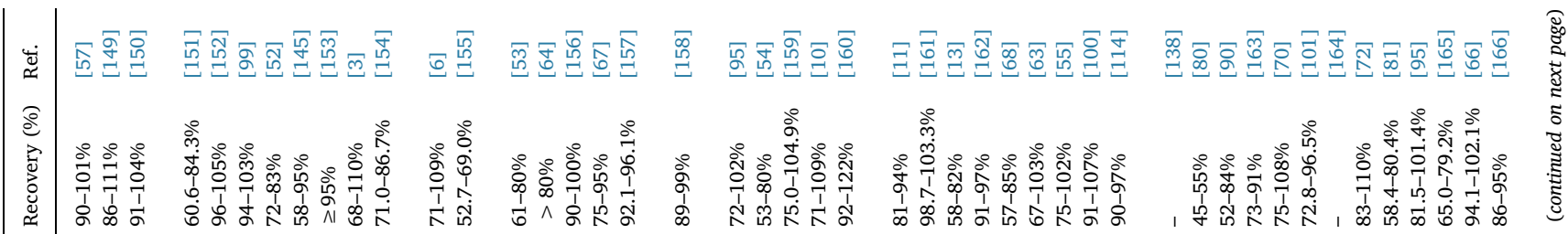

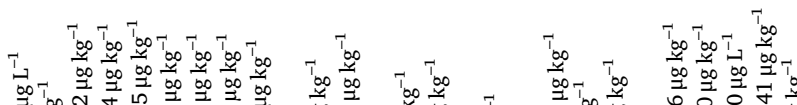

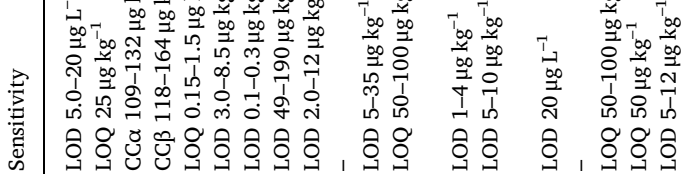

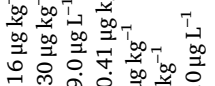
T

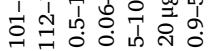

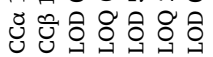

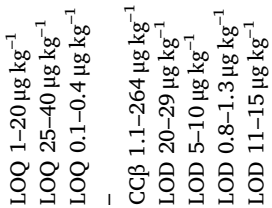

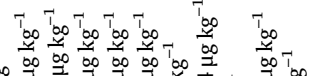

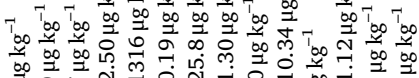
oo

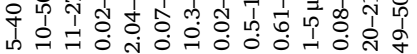

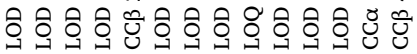

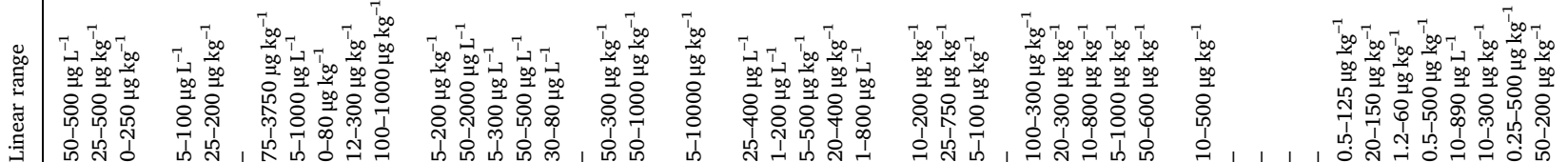

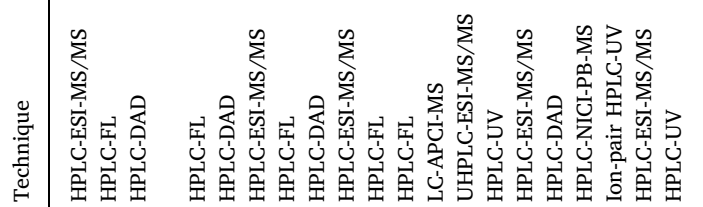

$\sum_{\substack{1 \\ 1}}^{\infty}$

$\sum_{i}^{\infty} \sum_{i}^{\infty} \sum_{i}^{\infty} \sum_{\infty}^{\infty} \sum_{i}^{\infty} \sum_{\infty}^{\infty} \sum_{i}^{\infty} \sum_{i}^{\infty} \sum_{i}^{\infty} \sum_{i}^{\infty} \sum_{i}^{\infty} \sum_{i}^{\infty} \sum_{i}^{\infty}$ $\sum_{i=1}^{\infty} \sum_{i}^{\infty} \sum_{i=1}^{\infty} \sum_{i=1}^{\infty} \sum_{i=1}^{\infty} \sum_{i=1}^{\infty}$

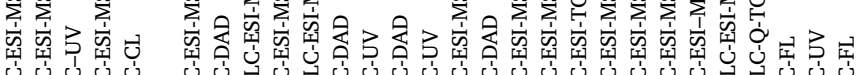
نे

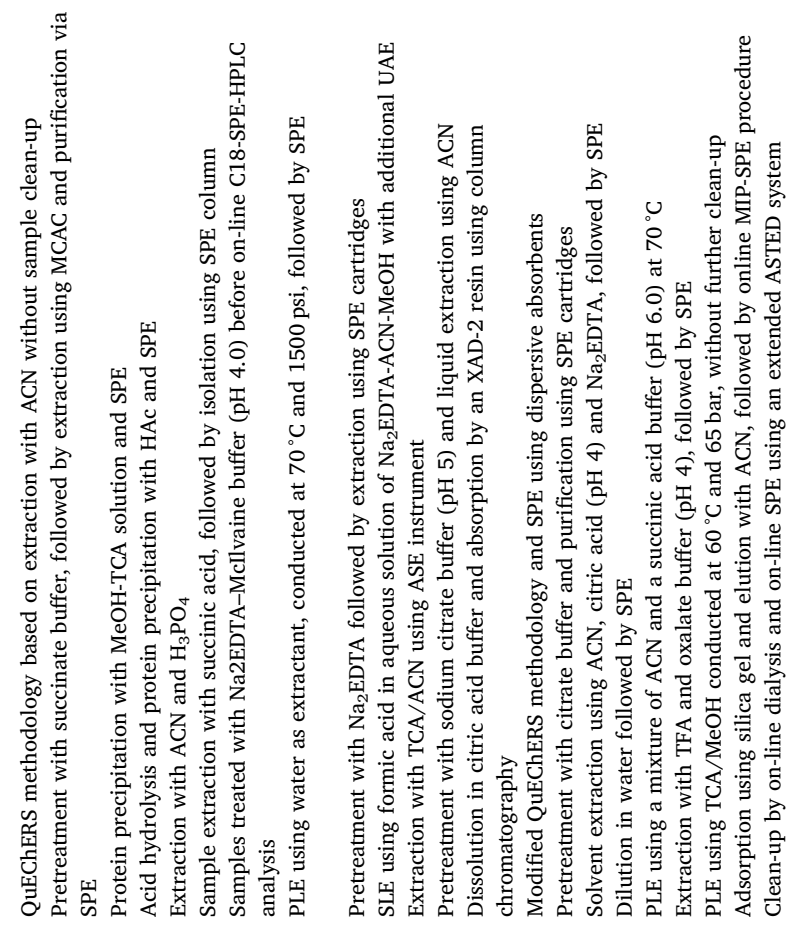

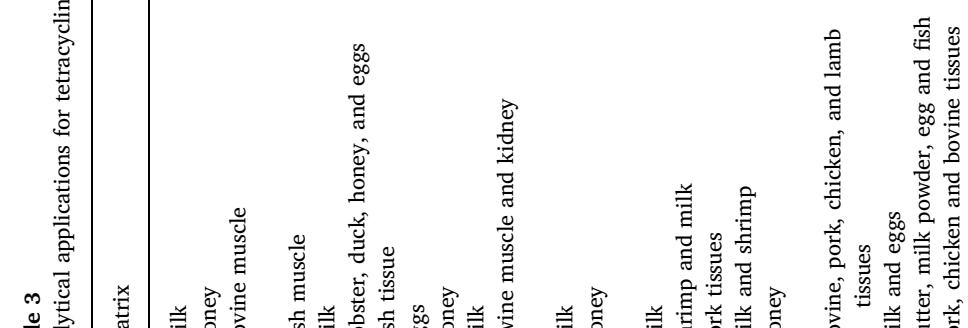

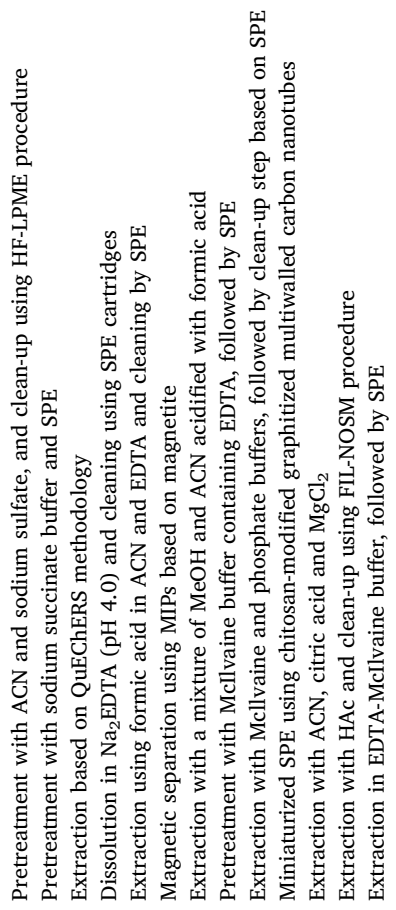

弟

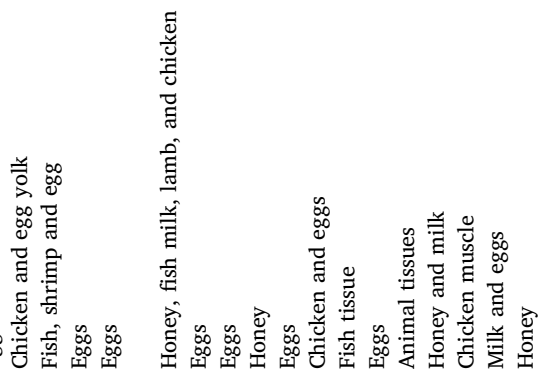




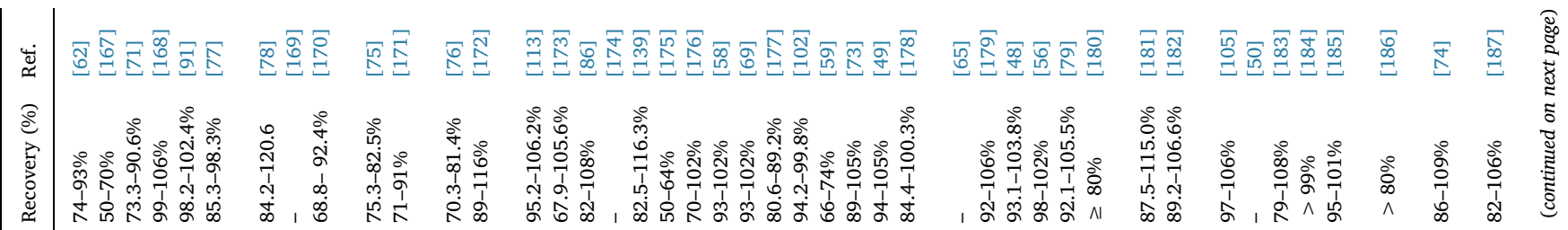

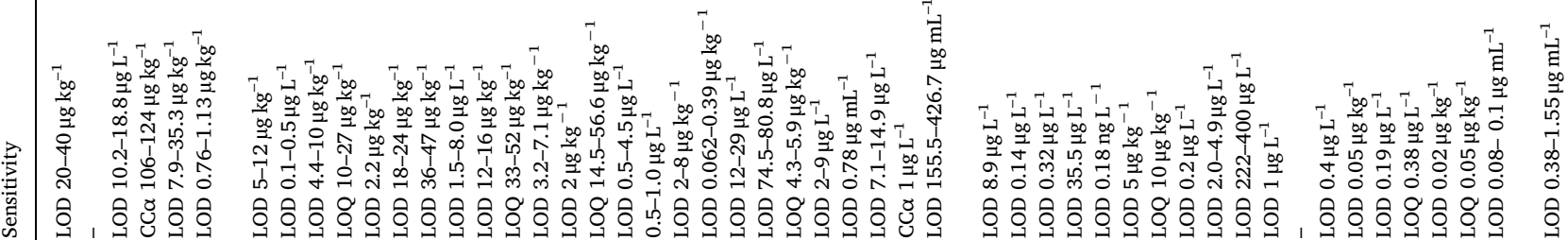

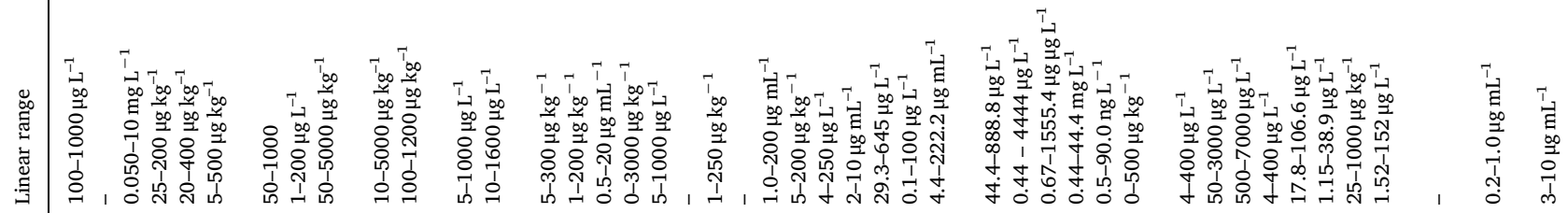

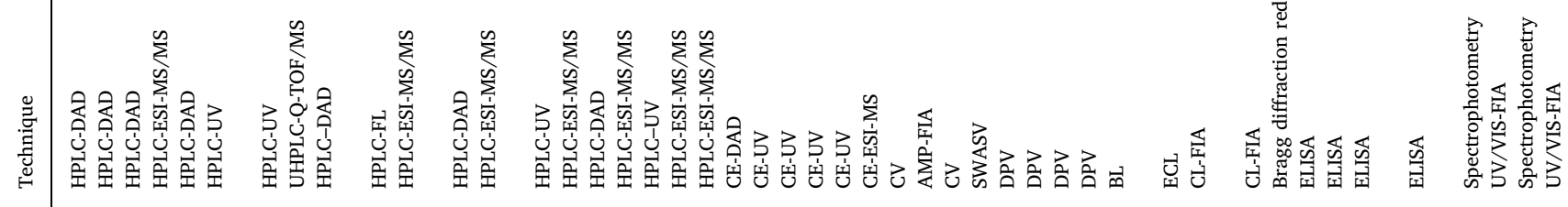

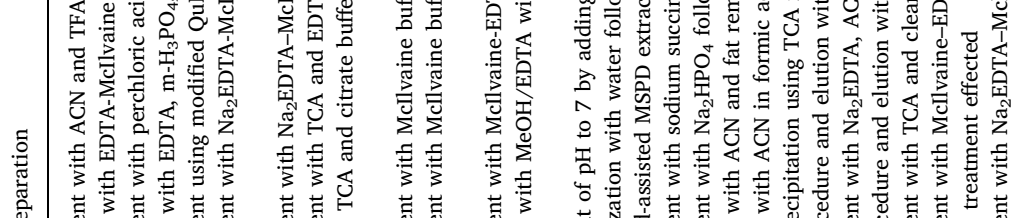

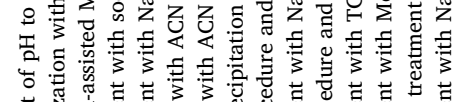

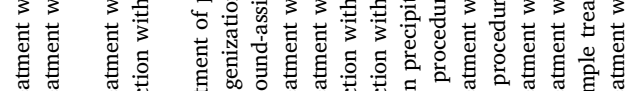

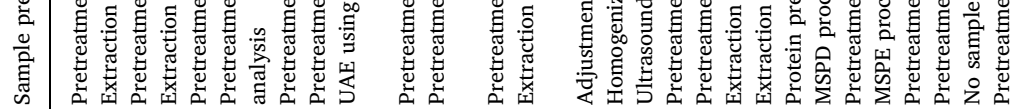

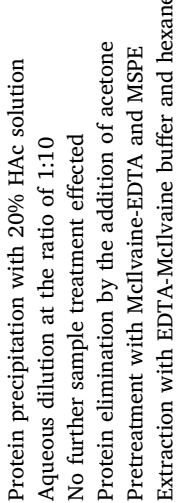

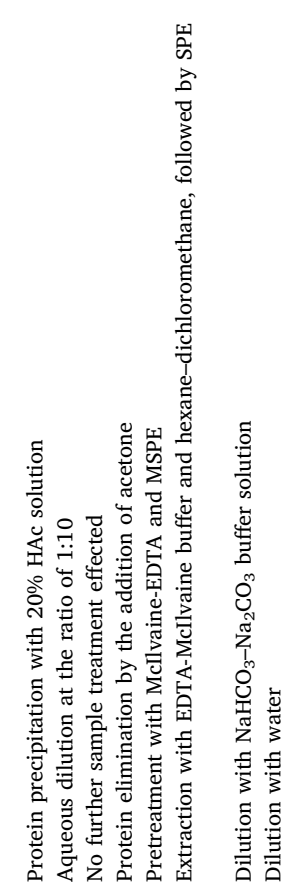

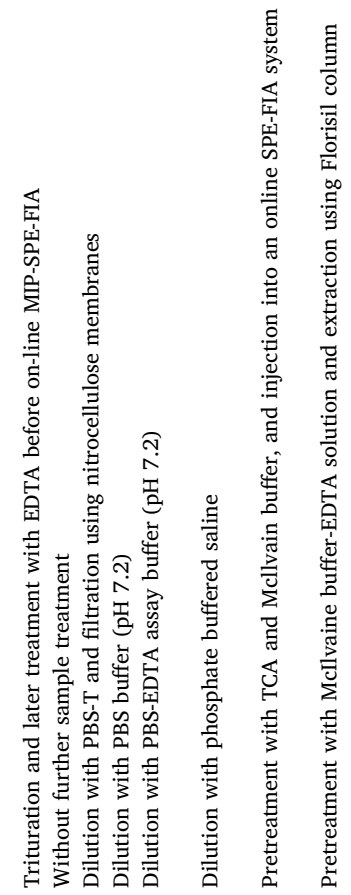

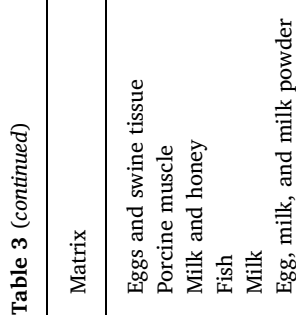

要<smiles>CCCCCCCC</smiles>

直

音

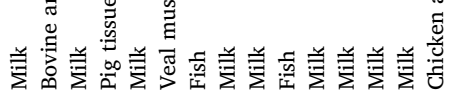

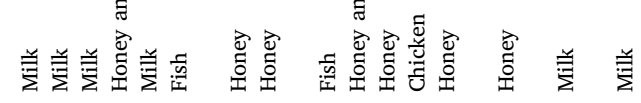


environment, these techniques are, to a greater extent, expensive and laborious, and are also characterized by automation difficulty $[82,83]$. In view of that, attention has been devoted to the development and implementation of simpler and eco-friendlier extraction methodologies [84].

\subsection{Ultrasound-assisted extraction}

Ultrasound-assisted extraction (UAE) technique is a mechanism involving solvent extraction with the aid of ultrasound to enhance extraction efficiency. The technique consists of shaking and heating the sample during the extraction process. Like the microwave-assisted extraction (MAE), UAE was developed as an alternative to Soxhlet extraction. The merits of the UAE are that it is relatively faster and requires smaller amounts of solvent $[47,85]$.

A point worthy of consideration when one seeks to obtain the maximum extraction efficiency and required selectivity in the employment of ultrasonication is the proper selection of the solvent type or solvent mixtures with water, with or without $\mathrm{pH}$ modification. Compared to other techniques, ultrasonification is more advantageous because the technique can be applied at room temperature, allowing the determination of thermolabile compounds. In addition, several extractions can be performed simultaneously and no expensive or specialized apparatus are required. One will note, however, that since the selectivity and enrichment capabilities of this technique are limited, further sample preparation steps are usually required to determine trace contaminants in foods $[12,47,85]$. By virtue of that, UAE is usually combined with other clean-up and/or preconcentration methodologies as an additional extraction process aiming at improving extraction efficiency.

A simple SLE procedure with $0.1 \%$ formic acid in aqueous solution of ethylenediaminetetraacetic acid (EDTA) $0.1 \%$ (w/v)-acetonitrile-metanol $(1: 1: 1, \mathrm{v} / \mathrm{v})$ was used for the extraction of tetracyclines from butter, fish tissue, milk powder and eggs [54]. An additional UAE step was, however, required to achieve recoveries within the $72-102 \%$ range by LC-MS/MS. The method was validated using matrix-matched calibration curves and the coefficient values determined indicated the absence of matrix effects.

Ultrasound-assisted matrix solid phase dispersion was applied for the determination of five tetracyclines using HPLC-DAD. This was the first attempt to apply matrix solid-phase dispersion combined with UAE for the preconcentration and clean-up of tetracycline residues from milk matrix. Interestingly, the process revealed high extraction yields [86]. Dispersive liquid-liquid microextraction has also been combined with UAE to isolate tetracyclines from egg supplement samples, followed by spectrophotometric determination using flow analysis. The additional ultrasonication step increased the method extraction capacity by approximately $6 \%$ [51]. These works, among others, have demonstrated the versatility of UAE when it comes to increasing tetracycline recovery in different foodstuffs by coupling with several sample extraction processes.

\subsection{QuEChERS}

QuEChERS (Quick, Easy, Cheap, Effective, Rugged, and Safe) method was introduced by Anastassiades et al. for pesticide residue analysis in vegetables and fruits. During its development, great emphasis was placed on obtaining a dynamic procedure that could be applied in any laboratory, given the simplicity and flexibility of the steps. The original QuEChERS methodology involves initial extraction with acetonitrile, followed by liquid-liquid partitioning promoted by the addition of magnesium sulfate $\left(\mathrm{MgSO}_{4}\right)$ and sodium chloride $(\mathrm{NaCl})$; and the supernatant is separated for further analysis. However, additional clean-up methodologies are commonly used for the removal of residual water and the isolation of analytes $[87,88]$.

In QuEChERS procedures, salts are added to promote the salting out-effect, which is commonly observed in several multi-residue 
Table 4

Detailed description of the advantages and drawbacks of selected sample preparation techniques for determination of tetracycline traces in food commodities.

\begin{tabular}{|c|c|}
\hline Techniques & Advantages \\
\hline SE & $\begin{array}{l}\text { Simplicity and availability of a solvent variety that provides } \\
\text { wide range of solubility and selectivity } \\
\text { High enrichment factors } \\
\text { Good recoveries }\end{array}$ \\
\hline \multirow[t]{2}{*}{ UAE } & $\begin{array}{l}\text { Improved extraction efficiency from the choice of an } \\
\text { appropriate solvent } \\
\text { No specialized equipment is required }\end{array}$ \\
\hline & $\begin{array}{l}\text { Inexpensive procedure that allows several extractions } \\
\text { simultaneously }\end{array}$ \\
\hline \multirow[t]{2}{*}{ QuEChERS } & $\begin{array}{l}\text { Robust method that presents simple, fast, and versatile step } \\
\text { Successful application in foods with different characteristics }\end{array}$ \\
\hline & Compatible with several analytical techniques \\
\hline SPE & $\begin{array}{l}\text { Commercial availability of a broad variety of sorbents and } \\
\text { simultaneous extraction of a wide range of organic analytes } \\
\text { No formation of emulsions } \\
\text { No decomposition of analytes sorbed onto the SPE cartridg } \\
\text { Easy configuration for different extraction modes } \\
\text { Automation possibility }\end{array}$ \\
\hline
\end{tabular}

MIP-SPE

MSPE

MSPD

PLE

SPME

DSPME

HF-LPME

DLLME
Cheap and easy preparation, and possibility of reuse High stability at extreme $\mathrm{pHs}$ and temperatures

High analyte retention and improved selectivity Cleaner sample extracts

Easy, fast and cheap handling

Convenient phase separation by an external magnetic field High extraction efficiency and suitability for extraction of volume large samples

Possibility of reusing magnetic adsorbents after an appropriate washing process

Low solvent consumption and no need of multiple extractions

Extraction selectivity can be achieved by packing a sorbent layer in the column bottom as an additional cleaning step

Automated sample handling

Faster extraction times and reduced solvent consumption

Elimination of the use of toxic solvents as extractants The use of high diffusion fluids improves the extraction process rate

Reduction of solvent consumption

Sampling and extraction are performed in a single step Ability to examine smaller sample sizes

Simple, economic and easy procedure Rapid mass transfer and shorter equilibrium time Enhanced extraction capacity, reduced time and solvent consumption decrease

High enrichment factors, relatively short extraction time, low cost of the extraction units and low consumption of toxic solvents

Excellent sample clean-up that helps to prevent carry-over problems

Addition of carrier agents in the sample facilitates the extraction of highly polar and hydrophilic compounds

Easy handling, quickness and low cost

Extraction occurs irrespective of time, and high preconcentration factors are achieved

Considerable reduction or even elimination of hazardous

solvent consumption via the use of IL as extracting solvents
Drawbacks

Consumption of large volumes of samples and organic solvents, which causes disposal problems

Partial extraction of samples with high affinity for water

Concentration of solvent impurities together with the sample

Possible formation of emulsions, which is time-consuming

Difficulty of automation and unsuitability for volatile analytes

Sample clean-up or analyte preconcentration steps are generally required

Extraction efficiency can be affected by the presence of water

Small amount of sample is obtained compared to other techniques Salt addition may cause spectral interferences in LWCC-based spectrophotometric techniques

Considerable solvent consumption

Time-consuming and cumbersome technique

Lack of selectivity

Extraction efficiency may be affected by poor packing

Batch-to-batch variation of the sorbents associated with low

recovery and high matrix interferences

Poor analyte fractionation

Selective extraction and clean-up limited to polar compounds Nonspecific hydrophobic interactions for application in aqueous media

Sometimes low recoveries associated with lack of reproducibility

Complexity and difficulty in synthesizing the magnetic materials Large sorbent amount and solution volume necessary for elution

Difficulty of automation and usually a further cleaning step is required

Extraction of many samples can be time-consuming

Expensive equipment and limited sample amount to be extracted The use of higher temperature of the solvent may decrease extraction selectivity, besides affecting stability of thermo-labile compounds

Variation of fiber coatings

Low robustness

Drag of matrix interferences affecting extraction of target analytes

Salt addition may impair sorbent dispersion in sample solution High $\mathrm{pH}$ values can affect analyte desorption

Extraction time depends on analyte transfer through the SLM, limiting the process speed

Need for commercially available equipment, which limits its application in routine laboratories

Manual preparation of extraction units affects recovery precision and makes procedure automation difficult

Matrix effects can be observed as the analytes are not isolated from the soluble coextractives

Automation difficulty due to the need to separate the phases 
Table 4 (continued)

\begin{tabular}{|c|c|c|c|}
\hline Techniques & Advantages & Drawbacks & Refs. \\
\hline \multirow[t]{7}{*}{$\begin{array}{l}\text { IL-mediated } \\
\text { extraction }\end{array}$} & Low cost, ease of synthesis, and operational simplicity & $\begin{array}{l}\text { Reactivity limited to certain reaction conditions and evidence of } \\
\text { side reactions during the desired reactions }\end{array}$ & \multirow[t]{7}{*}[66,125,188]{} \\
\hline & $\begin{array}{l}\text { High selectivity, precision and accuracy, and efficient sample } \\
\text { clean-up }\end{array}$ & Incompatibility with ion-exchange columns & \\
\hline & Application versatility & $\begin{array}{l}\text { Glassware and steel parts can be damaged by the produced HF } \\
\text { upon contact with fluoride-containing anions with moisture }\end{array}$ & \\
\hline & Compatibility with varied analytical techniques & $\begin{array}{l}\text { Some ILs show background interference in HPLC-UV analysis due } \\
\text { to the strong absorption in the UV region }\end{array}$ & \\
\hline & Organic solvent consumption elimination & & \\
\hline & Powerful solvents for chemical analysis & & \\
\hline & Environmentally friendly & & \\
\hline
\end{tabular}

methods. Depending on the nature of the solvent used in the partitioning step, good recovery percentages for polar analytes can be obtained, since the presence of salts in the medium decreases the solubility of these compounds in aqueous phase. This stage is seen to be very convenient in the extraction with acetonitrile because it does not dilute the sample extract and allows the separation of the organic and aqueous phases. Furthermore, the step involving water removal provides a final extract of low polarity, thus facilitating the precipitation of polar coextractives $[88,89]$. Since its inception, the method has been developed and modified, allowing the extraction of different compounds from several matrices.

A UHPLC-MS/MS multi-residue method for the selective determination of veterinary drugs in milk used a modified version of the QuEChERS methodology for sample preparation [10]. The extraction of pharmaceuticals, including doxycycline, was based on simple liquid extraction with acetonitrile without further clean-up step. Unlike the traditional method, acetic acid, $\mathrm{Na}_{2}$ EDTA and sodium acetate were also added to the extraction process. Fat removal and analyte extraction were performed in a single step devoid of the need for protein denaturalization before the extraction step. This type of modification is known as buffering QuEChERS. The use of UHPLC-MS/MS reduces analysis time and improves sensitivity and resolution. This technique allows the detection and quantification of several classes of veterinary drugs in less than $10 \mathrm{~min}$.

The combination of LC-MS/MS technique with different modifications of the QuEChERS procedure has been studied for the simultaneous determination of residues of tetracyclines and other veterinary drugs in egg samples $[11,90]$. A work reported in the literature used formic acid in acetonitrile-water containing EDTA as the extraction solution, followed by the evaluation of eight different sample cleaning schemes. To date, the best results for tetracyclines were achieved using HybridSPE cartridges based on zirconia-coated silica. An additional cleaning with dispersive sorbents was, nonetheless, necessary to obtain recoveries above $80 \%$. The latter step prolonged the time needed for sample preparation, providing clearer final extracts [11]. Other authors optimized a mixture of acetic acid in methanol-water as extracting solution. Two different clean-up procedures were tested for the removal of apolar substances and lipids from egg extracts; the procedures included dispersive-SPE using silica C18 and liquid-liquid extraction with n-hexane. The application of such procedures contributed toward the reduction of the matrix effect [90].

Dispersive solid-phase microextraction (DSPME) was also used as sample clean-up method following the application of the modified QuEChERS procedure for the analysis of tetracyclines in water and milk matrices using HPLC-DAD. Milk sample preparation was modified by adding $70-72 \%$ perchloric acid to the extracting mixture. Additionally, $14 \% \mathrm{NH}_{4} \mathrm{OH}$ solution was added to enable the alkalinization of the medium while analyte desorption was achieved using acetonitrile: perchloric acid: water mixture [91].

For the extraction of tetracycline via QuEChERS-like techniques, McIlvaine and/or EDTA buffer systems are often added to the extraction mixture to prevent chelation of these compounds with metal ions such as $\mathrm{Mg}^{2+}$ and $\mathrm{Ca}^{2+}$. EDTA acts as a competing chelating agent that facilitates the extraction of the analytes, thus paving the way towards obtaining high recoveries. In this sense, EDTA is commonly added to the extracting buffer and in many sample preparation steps. QuEChERS extraction is a fast and simple method that can be easily applied in routine laboratory $[90,91]$.

\subsection{Solid-phase extraction techniques}

Solid-phase extraction (SPE) is one of the most widely employed extraction techniques in sample clean-up and preconcentration procedures; it is used for the determination of tetracyclines in complex matrices [4]. It is a separation technique that involves solid-liquid partition equilibrium and is based on the separation mechanisms of low pressure liquid chromatography. Here, the extracting phase is a solid sorbent [92]. The technique was introduced in the early 1970s and became commercially available in 1978 in the form of disposable cartridges. A wide array of sorbents that rely on different mechanisms for analyte extraction/retention can be found available on the market [12].

Fig. 4 illustrates a typical SPE procedure, which involves the following steps: sorbent activation, sample percolation/sorption of the analytes in the solid sorbent, elimination of the matrix interferences (clean-up), and elution and concentration of the analytes for subsequent analysis using the most appropriate technique. Conditioning the solidphase materials entails passing organic solvents or water through the column so as to increase the effective surface area and reduce interferences. Sorbent drying is followed by the isolation and preconcentration of the analytes, which is accomplished by percolating the aqueous sample using a cartridge containing the sorbent. The analytes are initially retained for subsequent elution with another solvent in a collection tube [92]. The latter step is required owing to the disruption of the interactions between analytes and the solid-phase material; this step is accomplished by flushing small volumes of organic solvents, leading to desorption of the target analytes from the solid phase.
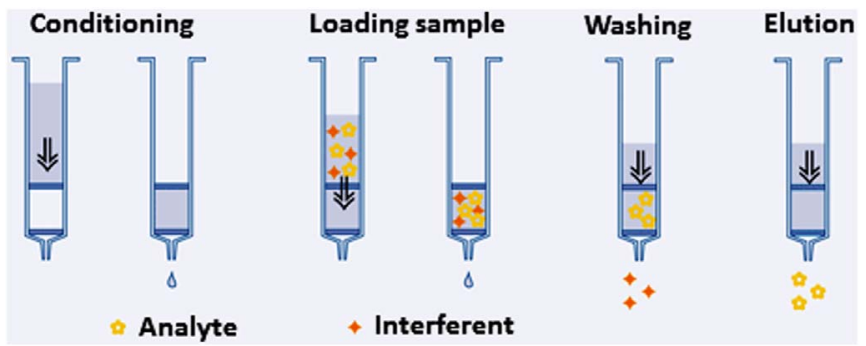

Fig. 4. Typical four-step SPE procedure. Conditioning: moisturising of the solid phase with an organic solvent or water; Sample loading: sample is pressed or drawn through the solid phase to enrich the analytes on the sorbent, so that the interfering components are not retained; Washing: remaining interfering components are washed from the sorbent; Elution: analyte is removed from the sorbent by eluting with a suitable solvent. 


\subsubsection{Sorbent materials in SPE}

Some of the parameters that ought to be considered when developing an SPE procedure include the choice of sorbent type and quantity, sample volume and solvent for cartridge activation, and elution of the analytes. Analyte characteristics, the nature of the matrix and its interferences should likewise be considered in the choice of sorbent [93].

There is a full range of reports in the literature describing the use of the SPE technique for the extraction of tetracycline residues from animal-derived foods [4], where OASIS HLB and C18 solid phases are found to be the most commonly used sorbents. OASIS HLB is a universal hydrophilic-lipophilic-balanced (HLB) polymeric reversed-phase sorbent that maintains its capability for greater retention owing to the fact that it is water-wettable. It is constituted by a specific ratio of two monomers, the hydrophilic $\mathrm{N}$-vinylpyrrolidone and the lipophilic divinylbenzene. C18 is based on octadecylsilane bonded to irregularly shaped silica particles; this nonendcapped sorbent is strongly nonpolar and nonselective and provides reversed-phase binding of hydrophobic compounds. Van der Waals forces, nonpolar, or secondary interactions such as hydrogen bonding to silica silanols can be combined to obtain analyte retention. In general, all these syringe-barrel-type cartridges are designed for use with vacuum manifolds and automated SPE instruments [92,94].

OASIS HLB polymeric cartridges were used for the extraction of tetracyclines, sulphonamides and chloramphenicol from bovine milk for further determination by HPLC-DAD [53]. Sample preparation involved protein precipitation through the addition of McIlvaine buffer and trichloroacetic acid in methanol, which yielded good recovery of tetracyclines. LC-MS/M technique was used to quantify veterinary drug residues, including tetracyclines, in eggs after treatment with sodium succinate buffer, followed by clean-up using the same type of cartridges [80]. In the aforementioned study, tetracycline extraction efficiency was affected by the use of OASIS cartridges; as such, the obtained recovery values were below those established by legislation.

Different authors have also studied the extraction of tetracycline residues from bovine milk using C18 SPE cartridges, where further determination was carried out by capillary electrophoresis (CE) with UV [59] and MS [73] detection. Field-amplified sample stacking was applied to the CE-UV method; this resulted in lower detection limits and better recoveries compared to the CE-MS method. The result may be attributed to the relatively greater sensitivity and specificity of the MS detector. Furthermore, egg samples were treated with acetonitrile, citric acid and EDTA for the extraction of tetracyclines and other veterinary drugs using C18 and OASIS HLB cartridges [23]. Here, the SPE step was required since the extract was redissolved in the mobile phase and a dirty solution was obtained. It is worth noting that, it was, however, impossible to inject the dirty solution into the UHPLC-MS/MS system, despite the fact that it was filtered. While the recovery results obtained with both cartridges were found to be similar for most analytes, the use of OASIS HLB yielded higher values (over 60\%) for tetracyclines.

By way of comparison, the use of OASIS HLB cartridges is highly recommended for the isolation and pre-concentration of tetracyclines from food samples such as milk and eggs. This is probably due to its stability $\mathrm{pH}$ values and in a broad spectrum of solvents, extraordinary retention of polar compounds, and a relative hydrophobic retention capacity found to be three-fold that of commonly used silica-based SPE sorbents-like C18 [92,94]. The number of articles published during the last two decades regarding tetracycline analysis in the study samples describing the use of OASIS HLB cartridges is much higher with respect to the use of $\mathrm{C} 18$ cartridges.

Carbograph 4 [95] and HybridSPE ${ }^{\mathrm{TM}}$ [11,55] cartridges and LiChrolut and Nexus SPE [63] cartridges have been used for cleaning animal-derived foods (milk, eggs and chicken tissue) in order to determine tetracycline residues by LC-MS/MS and HPLC-DAD, respectively. This class of antimicrobials and some veterinary medicinal products were also extracted and purified from milk samples using SPE columns based on Supelclean ${ }^{\mathrm{TM}}$ ENVI-Chrom P [64] and Florisil [96] sorbents aiming at determining drug residues by LC-PB-MS (liquid chromatography-particle beam-mass spectrometry) and spectrophotometry, respectively. All these works showed recovery and precision levels within acceptable limits according to the complexity of the food samples under analysis.

Remarkably, in spite of the drawbacks associated with SPE the importance of packing uniformity to avoid poor efficiency in complex matrices and the fact that the cartridges can only be used once, the technique has gained wider acceptance among researchers worldwide. This can be explained by the fact that the technique essentially prevents many of the problems associated with LLE, which include incomplete phase separations, low recoveries, the need for concentration of the extract and the use of expensive, breakable specialty glassware [94]. Some of the advantages associated with the use of SPE include less organic solvent usage, absence of foaming or emulsion problems, shorter sample preparation or minimal handling time which is tantamount to shorter analysis time, and clean sample extracts with high analyte recovery, even at low concentrations [92,97]. Furthermore, this technique presents ease of automation, extraction reproducibility, ability to increase analyte concentration in a selective manner and commercial availability of many SPE devices and sorbents, including the use of carbon-based nanomaterials [98], molecularly imprinted polymers (MIPs) [62,71,77,99,100], magnetic nanoparticles $[2,79,101,102]$, among others.

3.4.1.1. Molecularly imprinted polymers. Molecularly imprinted polymers (MIPs) are used as selective sorbent materials in traditional SPE; this has given rise to the moleculary imprinted polymer solidphase extraction (MIP-SPE) method [103]. MIPs are synthesized via polymerization of a functional monomer and a cross-linker around a target analyte, which acts as a molecular template. Thus, template-monomer complexes are obtained between a chosen template molecule and a complementary functional monomer. Fig. 5 illustrates the exact constellation of this coordination, which allows us to distinguish the different types of molecular imprinting technologies from one another. After the templates are removed through washing, cleavage of chemical bonds, or ligand exchange, the binding sites remaining in the resultant MIP can specifically capture the template molecule and its structurally related molecules. This is rendered possible because the imprinted sites contain a three-dimensional network presenting pores with the geometry and position of the functional groups complementary to those of the templates $[62,99,104,105]$. In view of that, these tailor-made materials can exhibit high affinity and selectivity towards a given target or group of target molecules.

To produce MIPs with desirable properties, the preparation method should be appropriately selected. Generally, the mechanisms involving MIP preparation include free-radical polymerization and sol-gel processes. The most commonly used free-radical polymerization methodology is bulk polymerization; this involves mechanical grinding and sieving steps to obtain small particles. The process is tedious, timeconsuming and needs a large amount of template molecules; as such, the obtained particles show a random shape and size. In an attempt to circumvent these shortcomings, more sophisticated and complex polymerization techniques have been proposed aiming at obtaining many different forms of MIPs, such as MIP particles, membranes, monoliths, and molecularly imprinted monolayers. Attractive polymerization methods for the preparation of MIP particles include suspension polymerization, emulsion polymerization, seed polymerization and precipitation polymerization $[99,104]$.

Precipitation polymerization has emerged as a desirable and scalable approach for the production of high-quality, uniform, and spherical imprinted particles $[106,107]$. The feasibility of preparing highly selective molecularly imprinting polymers using this method has recently been demonstrated [108-111]. Although a large amount of 


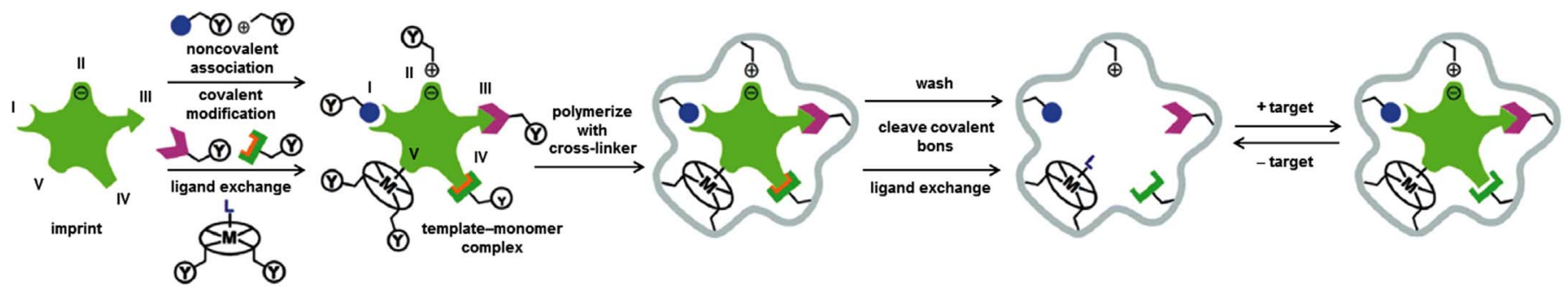

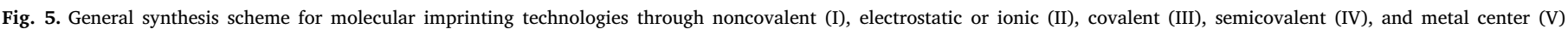

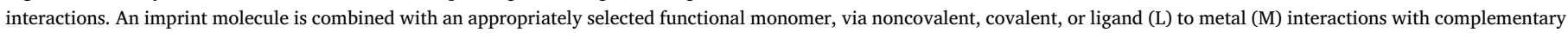

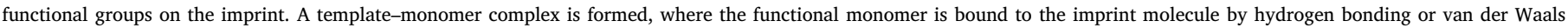

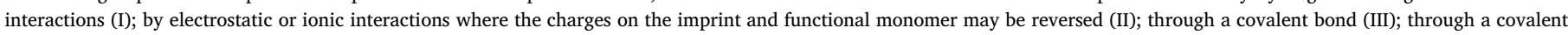

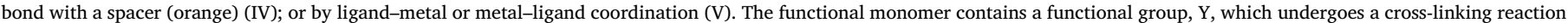
with an appropriate cross-linker (Reproduced from [104]).

template molecules is needed for the preparation process [99], precipitation polymerization has been the most outstanding method for the synthesis of MIPs focusing on the extraction of tetracycline residues from milk, eggs and other foods. Furthermore, by introducing the composite imprinting material strategy into sol-gel processes such as the preparation of nanomaterials and nanoimprinting, one is able to prepare MIPs with excellent interface characteristics and good electrical and optical properties. Clearly, this class of MIPs are more suitable for chemical or biological detection [104].

Several MIP-SPE methods combined with HPLC have been employed for determining tetracycline traces in food commodities. One of these methods made use of an imprinted polymer synthesized by precipitation polymerization for application in milk, egg and pork samples. Chlortetracycline was used as template molecule and methacrylic acid as functional monomer. Samples were initially treated with acetonitrile and trifluoroacetic acid [62]. The imprinted monolithic columns were also prepared via in situ molecular imprinting technique using tetracycline as the template. In this case, the functional monomer consisted of methacrylic acid and the cross-linker of ethylene glycol dimethacrylate. In addition, cyclohexanol and dodecanol were used as the mixed porogenic solvents. Thereafter, MIP-SPE coupled with C18 column was used to determine tetracyclines in milk and honey samples after treatment with $\mathrm{HClO}_{4}$ solution [71]. Both methods were able to selectively extract the target substances.

A noteworthy application of molecular imprinting technique for selective tetracycline extraction in foodstuffs (duck, lobster, egg, and honey) was reported by Jing et al. [99]. The use of tetracycline as the template, methacrylic acid as a functional monomer, trimethylolpropane trimethylacrylate as a cross-linker, 2,2-azobisisobutyronitrile as a free radical initiator, and a methanol and acetonitrile mixture as solvent allowed the synthesis of new MIPs to be used as SPE sorbents. The synthesized MIP-SPE showed better clean-up efficiency and a higher recovery compared to the commercial sorbents C18 and Oasis HLB cartridges, respectively.

These results evidently demonstrate that the use of MIPs as sorbent materials contributes to a more efficient preconcentration of tetracycline residues and removal of the interfering compounds from food matrices. Their use enables a much more selective extraction of the target antibiotics than conventional SPE sorbents. Moreover, these restricted access materials are stable under harsh chemical conditions such as extreme $\mathrm{pH}$, high pressure and high temperature, and can be reused dozens of times [104].

3.4.1.2. Magnetic sorbents. Magnetic solid phase extraction (MSPE) was developed during the past decade; it is a magnetically assisted chemical separation method that uses magnetic-nanoparticle-based support as absorbent material. This method shares the basic principles of the conventional SPE technique. Magnetic nanoparticles are superparamagnetic, i.e., they are attracted to a magnetic field, yet no residual magnetism is retained after field removal. Magnetic sorbents are commonly prepared by organic or inorganic polymerization in the presence of magnetite $[2,112]$.

An attractive property of this technique is that magnetic adsorbents can be isolated very quickly from the sample matrix by applying an external magnetic field, so that the superparamagnetic particles will not agglomerate upon the removal of the field. In this procedure, after the dispersion of magnetic support in the sample solution and formation of the suspension, the analytes adsorbed by the magnetic support are collected using a magnet without additional centrifugation or filtration. Finally, the analytes are consequently eluted from the recovered adsorbent and the extract is subjected to analysis $[2,102]$. An outstanding advantage of this technique lies in the possibility of improving the extraction performance by means of a high ratio between surface area and extraction phase volume.

In order to determine tetracycline residues by capillary electrophoresis, milk samples were prepared by MSPE using a paramagnetic, phenyl-functionalized silica adsorbent [102]. The magnetic phenyl silica adsorbents covered with previously synthesized magnetite were obtained via emulsion polymerization by varying the molar ratio of phenyltrimethylsilane and tetramethylorthosilicate; these adsorbents were evaluated in terms of their $\mathrm{pH}$ and degree of hydrophobicity for analyte retention.

MSPE based on magnetic silica-coated materials was also used for tetracycline preconcentration in milk samples [2]. The magnetic materials were synthesized by polymerization from previously prepared magnetite, tetramethoxysilane, Triton X-100 and cetyltrimethylammonium bromide (CTAB) using metanol as solvent. The method consisted of magnetic support dispersion onto untreated milk samples. The tetracyclines desorbed with acidified methanol from the magnetic support were determined simultaneously by spectrophotometic flow injection analysis.

Milk samples were also prepared by MSPE using b-cyclodextrin functionalized magnetic graphene oxide nanoparticles (b-CD-MGONPs) [79] and a laccaic acid-loaded magnetite nanocomposite [113] for the extraction and preconcentration of sought substances. The extracted tetracyclines were analyzed by differential pulse voltammetry and HPLC-UV, respectively. The evaluation of both extraction schemes showed that the electrochemical method was comparatively more sensitive, whereas the chromatographic method exhibited a better extraction performance.

The functionalization of MIPs with magnetic materials has also been used for the separation of tetracycline antibiotics from foods such as egg and chicken tissue samples. In the preparation of the magnetic MIPs, hydrophobic $\mathrm{Fe}_{3} \mathrm{O}_{4}$ magnetite was used as the magnetically susceptible component, oxytetracycline as the template molecule, methacrylic acid as the functional monomer, and styrene and divinylbenzene as the polymeric matrix components. Following the magnetic isolation and desorption of the analytes, the residues were detected and quantified by LC-MS/MS [101].

MSPE has attracted considerable attention in recent years because 
of its biological applications in cell isolation, enzyme immobilization, protein separation, and analytical applications related to the pre-concentration of organic compounds from large volume samples, including food matrices [79,102]. Compared to conventional pretreatment methods, the magnetically assisted separation is easier, faster, cheaper and environmental friendlier [2]. Furthermore, MSPE overcomes the problem of column packing by simply using an external magnetic field [113]. This type of technology for complex sample preparation has aroused great interest, since it provides improved efficiency in the extraction of target analytes [112].

\subsubsection{Automated SPE}

Over the last years, on-line and automated SPE have gained a great deal of popularity and have been recommended for chemical analysis. Automated SPE systems include on-line SPE with measurement of the eluate and SPE with direct on-column measurement. In general, the analyte is retained by the solid support, and once eluted with an appropriate solvent, it can be measured in the eluate or directly using the support. Although the configuration of the latter is not very common in analytical chemistry, it provides good detection limits because of the avoidance of the dilution phenomenon involved in the elution process [92]. On the other hand, most on-line SPE protocols for the determination of tetracyclines in animal-derived foods consist of SPE columns coupled with one of the following: HPLC systems [76-78,100,114], spectrophotometric detectors [74] or chemiluminescence [105] detectors.

To date, HPLC coupled with sample pre-treatment using SPE has been the on-line configuration most frequently used for tetracycline analysis in foodstuffs. Tetracyclines were determined in egg and honey samples by coupling HPLC with on-line SPE using PLRP-S [114] and C18 [78] columns, respectively. In addition, on-line SPE based on MIP coupled to HPLC-UV has also been used to determine tetracycline antibiotic residues in egg samples [100], and egg, milk, and milk powder samples [77]. All tested sorbents showed sufficient sensitivity for residue detection in the target matrices, achieving good recoveries in each case.

Essentially, there has been a clear trend toward the use of automation techniques that merge sample pre-treatment using on-line SPE with liquid chromatography separation. The coupling technique improves the accuracy and reproducibility of the chromatographic method. Furthermore, the sensitivity of on-line SPE-HPLC method is much better, in that the whole sample extract is transferred to the analytical column. In conventional off-line techniques, only a small portion of the extract is injected [92].

Apart from the on-line HPLC-SPE procedures, tetracyclines have also been determinated in fish samples by coupling MIP-SPE with chemiluminescence flow injection analysis (FIA). Here, polytetrafluoroethylene (PTFE) tubes were packed with MIP and were connected to the FIA system injection valve sampling loop. The chemiluminescence intensity was found to be linear in the application range of the sample. The combination of these procedures resulted in a new flow method capable of on-line extraction and determination of tetracycline residues in foods [105].

Another flow injection method worth mentioning is the one associated with on-line tetracycline preconcentration from milk using SPE and subsequent spectrophotometric determination [74]. The extraction minicolumn is packed with C18 sorbent material. After treatment with trichloroacetic acid and Mcllvain buffer, the samples are injected into the flow system and the tetracyclines adsorbed on the C18 resin are desorbed by a stream of eluent (methanol-buffer solution, 40:60, v/v). Here, the tetracyclines are conducted to the detector flow cell through the eluent. The analytical frequency of the flow system of six samples per hour is found to be very low relative to other FIA methods that can analyze between 42 [31] and 52 [36] samples per hour.

Remarkably, on-line SPE is best recommended because it offers several advantages, including a reduction in the sample pretreatment time, and thus, an increase in the sample throughput. This technique allows automated conditioning, washing, and elution processes. In addition, it can reduce the risks of sample contamination, as well as analyte degradation and loss by evaporation. Cartridges used in on-line SPE can usually be reused; this helps to reduce the amount of solvent consumed and the costs involved in organic solvent waste disposal [92].

\subsection{Matrix solid-phase dispersion}

Matrix solid-phase dispersion (MSPD) is a widely applied sample preparation procedure for extracting/purifying analytes from a variety of solid, semi-solid or highly viscous foodstuffs [12]. This technique consists of mechanical blending of the matrix onto a solid support to promote its complete disruption, allowing the interactions of the sample matrix with a solid support bonded-phase and the subsequent extraction of target analytes using a suitable elution solvent. MSPD is primarily used due to its simplicity, flexibility, selectivity, and the possibility of performing extraction and clean-up in a single step, leading to a more rapid pre-treatment and lower solvent consumption [115-117].

Over the last decade, MSPD procedures have been little reported for the extraction of tetracyclines in foodstuffs. Interestingly, a matrix solid-phase dispersion-capillary electrophoresis (MSPD-CE) method was developed by $\mathrm{Mu}$ et al. for the simultaneous determination of tetracyclines in milk. The proposed MSPD proved to be a simple and effective method for sample pretreatment. In that work, several solid support materials were investigated; and silica with bonded C18 chains was selected as the best sorbent [69]. Other authors proposed ultrasound-assisted dispersive extraction of tetracycline residues from milk samples with the determination performed by HPLC-DAD. Among the tested sorbents, Bond Elut Plexa was found to be the most efficient for the extraction of the analytes as it presented the highest recovery. The reliability of the method was additionally checked by the analysis of a bovine milk certified reference material [86].

\subsection{Pressurized liquid extraction}

Pressurized liquid extraction (PLE) uses conventional solvents at elevated pressures and temperatures that do not reach their critical point to extract solid samples more quickly [118]. In general, a solid sample is packed into a stainless steel extraction cell and extracted with a suitable solvent under elevated temperature $\left(40-200^{\circ} \mathrm{C}\right)$ and pressure (500-3000 psi) conditions for short periods (5-15 min). Finally, the sample extract is purged into a collection vial with the aid of compressed gas [119]. The use of solvents at temperatures higher than their atmospheric boiling point improves solubility of the analyte. Furthermore, it also enhances the mass transfer properties of the analyte, which in turn improves the extraction yields due to improved sample wetting, better solvent penetration and higher diffusion rates $[120,121]$.

A multiclass UHPLC-MS/MS method was developed and validated for the analysis of different antimicrobial agents in eggs by combining a PLE technique for target compound extraction. Tetracyclines were extracted using a 1:1 mixture of acetonitrile and a succinic acid buffer $\mathrm{pH}$ 6.0) at $70{ }^{\circ} \mathrm{C}$ [68]. Liu et al. also reported to have used PLE to extract tetracyline drugs from egg, fish and shrimp samples with the aid of HPLC-UV for residue determination. The extraction procedure employed a trichloracetic acid/methanol mixture set at elevated temperature $\left(60^{\circ} \mathrm{C}\right)$ and pressure (65 bar) [55]. While both methods were effective in accomplishing their goal, the latter appears to have attained better results than the former when one considers the limits of detection and recovery values obtained in each case. Here, an opposite result was expected, since the HPLC-MS/MS technique is relatively more sensitive and specific compared to the HPLC-UV. Thus, a greater efficiency of PLE procedure is observed in the latter compared to the former. 


\subsection{Microextraction techniques}

Microextraction techniques evolved from the classic sample preparation procedures and basically consist of the miniaturization of the latter. These techniques were developed to address the following concerns: limited selectivity, incompatibility with instrumental techniques, the need to extract analyte traces from a large number of samples, the quest for the reduction of costs and time of analyses and for minimizing the consumption of hazardous organic solvents that pose serious environmental and public health problem [122].

These techniques rely on quantitative yet non-exhaustive transfer of analytes through the equilibration of small portions of adsorbents or sorbents and large amounts of samples in direct or indirect contact [123]. Solid-phase microextraction (SPME) and liquid-phase microextraction (LPME) are microextraction techniques that have arisen as a response to the drawbacks faced by traditional techniques [122]. Each miniaturized technique for tetracycline extraction presents its peculiarities, which are described in detail below.

\subsubsection{Solid-phase microextraction}

Solid phase microextraction (SPME) is a solvent-free sample preparation technique and consists of the miniaturizacion of the traditional SPE technique. In SPME, the analytes are sorbed onto the surface of silica fiber coated with an appropriate stationary phase. This technique is controlled by partition equilibrium and may be applied to gaseous (headspace vapor), liquid and solid samples. While the extraction maximum sensitivity is obtained at the equilibrium point, it is not necessary to reach this point to obtain good results. Thus, the extractions can be performed for a defined period of time $[47,124]$.

The basic procedure for SPME involves two essential steps. The first step involves the partitioning of analytes between the sample matrix and the sorbent coating. This step is followed by desorption of the analytes into the suitable instrument mobile phase for separation, usually performed by GC or HPLC. In SPME-GC analysis, the analytes are thermally desorbed into the injector of the chromatograph; this is generally restricted to volatile and thermally stable compounds. Moreover, sample matrix derivatization is required for some applications. In the case of SPME-HPLC, desorption is carried out in an appropriate interface using a static or dynamic mode. The interface consists of six-port injector with a special fiber desorption chamber installed in place of sample loop. Here, desorption is carried out by means of an organic solvent or mobile phase. This is attributed to the fact that thermal desorption at high temperature promotes the degradation of the polymer and incomplete desorption of many nonvolatile compounds from the fiber. Nevertheless, in-tube SPME devices are generally more suitable for LC automation [47,124,125].

SPME matched with GC has been widely applied for metabolite analysis in plant extracts, food, environmental and biological samples. Research and development for implementation of this microextraction technique have been conducted in biomedical, botanical, pharmaceutical and food sciences [126]. By contrast, a review conducted by Ridgway et al. summarizes some automated in-tube SPME applications combined with HPLC using diode array (DAD) and mass spectrometry (MS) detector for analysis of endocrine disruptors and heterocyclic amines, respectively, in different foods [47].

A miniaturized SPE method was used for the preconcentration of tetracycline antibiotics in honey and milk using extraction sorbent based on chitosan-modified graphitized multiwalled carbon nanotubes (G-MWNTs) [98]. The analytes were extracted by passing the samples directly through the cartridges, using chitosan-modified G-MWNTs as the solid sorbent and a mixture of acetonitrile/acetic acid as the eluent in miniaturized SPE. Some variables, such as the amount of sorbent, elution solutions, and carbon nanotube types, were optimized in this procedure. Residues were detected by ultra high performance liquid chromatography and quadrupole time-of-flight mass spectrometry (UHPLC-Q-TOF/MS). The new synthesized sorbent showed excellent recoveries and a relatively strong adsorption and enrichment capacity toward tetracyclines.

The applicability of SPME to a broader array of analytes and matrices has been expanded by devoting much attention and efforts in the engineering of new sorbent materials. In this sense, ionic liquids (ILs) can be structurally designed aiming at a selective extraction of target analytes based on unique molecular interactions according to their unique physico-chemical properties. Considerable benefits can be gained by exploring this feature for SPME experiments, since selective extractions can be achieved by adding certain substituents into the structural make-up of these compounds. Thus, the application of ionic liquids as innovative sorbent materials for SPME contributes toward expanding the field of research [125].

3.7.1.1. Dispersive solid-phase microextraction. Dispersive solid-phase microextraction (DSPME) is based on a concept similar to those of SPME and stir bar sorptive extraction (SBSE) methodologies. Instead of the column-like format, dispersive solid-phase sorbent particles are used to trap analytes for analysis [127,128]. Like the other aforementioned techniques, SBSE is another sorptive technique developed with the aim of overcoming the limited extraction capacity of SPME fibers. A glass stirrer bar is coated with a potentially thick bonded absorbent layer (PDMS) meant to provide a large stationary phase surface area; this yields a higher phase ratio (50-250 times greater than that of SPME), resulting in a better extraction capacity and high recovery. The analytes are transferred from the bar by thermal desorption with the aid of gas chromatography, or solvent elution for liquid chromatography [47,129].

In DSPME, the extraction and enrichment of the analytes is accomplished with the addition of only a small amount of dispersive solid sorbent to sample solution. After extraction, the sorbents containing the retained analytes are settled by centrifugation or filtration. They are then washed with a small volume of solvent for analyte desorption, discarding most of the liquid followed by drying. In this type of procedure, the target analytes fully interact more easily with the adsorbents; hence, the adsorption capacities obtained in this method are higher than in conventional SPE. Since its discovery, DSPME has been recognized as a promising sample preparation technique, though it has been little implemented in food analyses [128].

DSPME in combination with HPLC-DAD was applied for tetracycline determination in water and milk samples [91]. Milk samples were prepared using a modified QuEChERS method. Several silica-based and polymeric sorbents were assessed in their adsorption capacities to preconcentrate the analytes in the dispersive format from aqueous or organic solutions. The effects of various experimental parameters on the performance of the method were also investigated. Silica-based sorbents functionalized with amine or carbonyl groups presented the highest tetracycline adsorption under organic environment. In this procedure, the analytes were adsorbed in a small amount of the optimized dispersive sorbent and subsequently desorbed with a small volume of desorption solution for further analysis by HPLC. The sorbent functionalization here showed better capabilities for trapping tetracyclines than those of polymeric sorbents in the dispersive format under solvent-enhanced environment [127].

DSPME is superior to traditional SPE in terms of enhanced recovery, extraction time-reduction, solvent consumption minimization and protocol simplicity. In addition, the performance of DSPME processes can be improved considerably by selecting sorbents according to their advantages such as the presence of reaction centers and a high surface area [130]. Thus, the application of novel reactive and non-toxic nanostructure adsorbents that ensure a high surface area and reactive centers can improve the extraction capacity of the method.

\subsubsection{Liquid-phase microextraction}

Liquid-phase microextraction (LPME) emerged from the need to apply small volumes of liquids for analytical extractions; it is, in 
essence, a miniaturized implementation of conventional liquid-liquid extraction [131,132]. This technique is based on the distribution of analytes between a small amount of an appropriate solvent and an aqueous phase containing these analytes. Research in this field was initiated in 1996 with the use of organic droplets suspended from the tip of a microsyringe, and the technique was named single drop microextraction (SDME). Later on, the technology was refined by implementing the use of porous hollow fibers for the protection of the extraction liquids. This gave rise to the development of hollow fiber liquid phase microextraction (HF-LPME) in 1999. In 2006, Rezaee et al. developed the dispersive liquid-liquid microextraction (DLLME) which represented an advancement in miniaturized liquid-liquid extraction $[133,134]$. The last two techniques have gained prominence in the analysis of tetracycline residues in animal-derived foods.

3.7.2.1. Hollow fiber membrane microextraction. Hollow fiber liquid phase microextraction (HF-LPME), also known as hollow fibersupported liquid membrane (HFSLM), is a technique that uses a porous hollow cylindrical fiber typically made of polypropylene to immobilize organic solvent in pores [133,135]. A membrane represents a selective barrier between a donor phase and an acceptor phase, through which a certain amount of mass can be transferred from one phase to another. The membrane supported separation occurs thanks to the capacity of the membrane to transport some components from the donor phase to the acceptor phase faster than in other techniques; this process is also referred to as permeation [136]. In this technique, the extraction takes place inside the lumen of the polypropylene porous hollow fiber, which contains the microvolume of the extracting liquid. This ensures that the extracting liquid is not in direct contact with the sample solution [85].

In practical terms, the pores of a porous hollow fiber are impregnated with an organic solvent to produce immobilization of the solvent into the pores of the hollow fiber. Thus, a thin layer of organic solvent is formed inside the wall of the hollow fiber. The next step consists of filling the lumen of the hollow fiber with microliters of an appropriate acceptor solution (aqueous or organic phase). Finally, the hollow fiber is immersed into the aqueous sample (donor phase) containing the compounds of interest, without direct contact between the acceptor phase and the sample. To expedite the extraction, the sample can be extensively heated, agitated or stirred. The analytes are then extracted from the aqueous sample (donor phase) through the organic layer on the walls of the hollow fiber and then placed into an acceptor phase inside the lumen of the hollow fiber [85,133,135]. Fig. 6 illustrates a general scheme of the HF-LPME procedure.

HF-LPME is controlled by partition equilibrium and may be employed both in two-phase or three-phase mode (Fig. 7), according to the type of acceptor phase and the characteristics of the analytes to be extracted. In two-phase systems, the acceptor solution is the same organic solvent immobilized in the hollow fiber pores. This mode is usually used to extract hydrophobic compounds, i.e., analytes with high solubility in an organic solvent immiscible with water. However, in a three-phase mode, the acceptor solution is another aqueous phase with controlled $\mathrm{pH}$. This extraction mode is limited to basic or acidic analytes with ionizable functions. In this HF-LPME configuration, the analytes must be in their non-ionized form in the donor solution to facilite their dissolution in the thin film of organic solvent contained in the hollow fiber pores. The analytes are, thus, transferred into the aqueous acceptor phase. The acceptor phase is composed of an acidic or alkaline aqueous solution and must have a $\mathrm{pH}$ value opposite to that of the donor phase to favor analyte ionization. In this sense, one is able to prevent the possibility of the analytes returning to the organic phase and thus to the donor solution, favoring their extraction. In this method, the $\mathrm{pH}$ adjustment plays a central role $[85,133,135]$.

Among the parameters that can affect the extraction efficiency of HF-LPME include type of hollow fiber materials, type and volume of organic solvent, $\mathrm{pH}$ of donor and acceptor phases, extraction time,

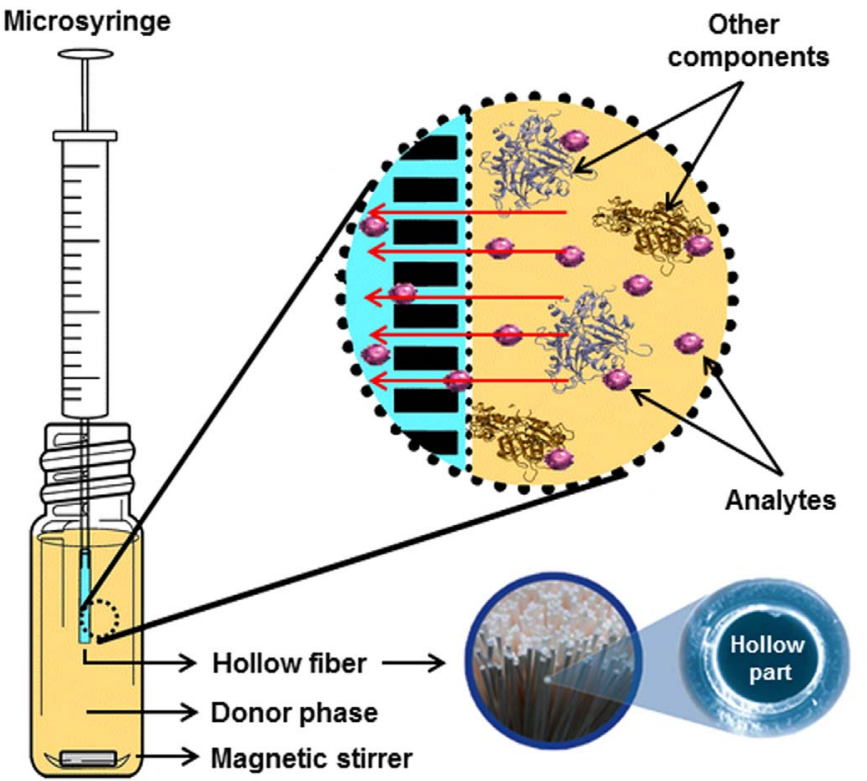

Fig. 6. Schematic illustration of the HF-LPME procedure.

temperature, stirring speed, and ion strength due to salt addition [135]. Carrier-mediated LPME is used as an active transport mode in order to promote the extraction of hydrophilic compounds from the donor aqueous phase into the organic phase [137]. The three-phase mode is the most commonly used configuration of HF-LPME because it is relatively easier to clean. In general, three-phase HF-LPME has been applied for the extraction and determination of analytes from complex matrices such as biological and food samples [138].

An efficient hollow fiber-based preconcentration method using carrier-mediated three-phase LPME prior to HPLC-UV was developed for simultaneous extraction and determination of tetracycline antibiotics from bovine milk, human plasma and water samples [139]. Milk samples are first pretreated with $\mathrm{Na}_{2} \mathrm{HPO}_{4}$. The effects of compositions of the aqueous sample and acceptor phase, type and volume of organic solvent, stirring rate and extraction time are evaluated in order to obtain high extraction efficiency. All the extractions are carried out using an Accurel Q3/2 polypropylene hollow fiber membrane. The carrier composed of a solution of 1-octanol containing $10 \%(\mathrm{w} / \mathrm{v})$ of Aliquat336 is immobilized in the pores of a hollow fiber. The aqueous acceptor phase, consisting of $0.1 \mathrm{~mol} \mathrm{~L}^{-1} \mathrm{H}_{3} \mathrm{PO}_{4}$ and $1.0 \mathrm{~mol} \mathrm{~L}^{-1} \mathrm{NaCl}$ with $\mathrm{pH}=$ 1.6 , is placed inside the lumen of the porous hollow fiber. The fiber is then immersed into the aqueous sample for subsequent tetracycline extraction. After this process, the aqueous extract obtained is directly injected into the HPLC system. The active transport mode of analytes allows a simple extraction setup with high clean-up effect and high enrichment factors as well as good precision.

Another technique that deserves mentioning is that proposed by Tajabadi et al. which combines HF-LPME with HPLC-DAD for the determination of tetracycline residues and other antibacterials in food samples [138]. Before the extraction procedure, all samples are adequately prepared to obtain an aqueous solution. The precipitation of proteins from milk sample is achieved by adding $\mathrm{K}_{3}\left[\mathrm{Fe}(\mathrm{CN})_{6}\right] \cdot 3 \mathrm{H}_{2} \mathrm{O}$ and $\mathrm{Zn}\left(\mathrm{CH}_{3} \mathrm{COO}\right)_{2} \cdot 2 \mathrm{H}_{2} \mathrm{O}$. The performance of the method is optimized by allowing the parameters to affect extraction using a Box-Behnken design under response surface methodology. HF is impregnated with $10 \%$ Aliquat-336 in 1-octanol to fill the pores with the solvent while the aqueous acceptor solution consisting of $1 \mathrm{~mol} \mathrm{~L}^{-1} \mathrm{NaOH}$ and $\mathrm{NaCl}$ is introduced into the lumen of the HF. Once the extraction procedure is completed, the analyte-enriched acceptor phase is analyzed using the HPLC instrument. The authors applied a multivariate curve resolutionalternative least squares (MCR-ALS) algorithm to solve the problem related to some overlapped peaks in multivariate second-order data of 


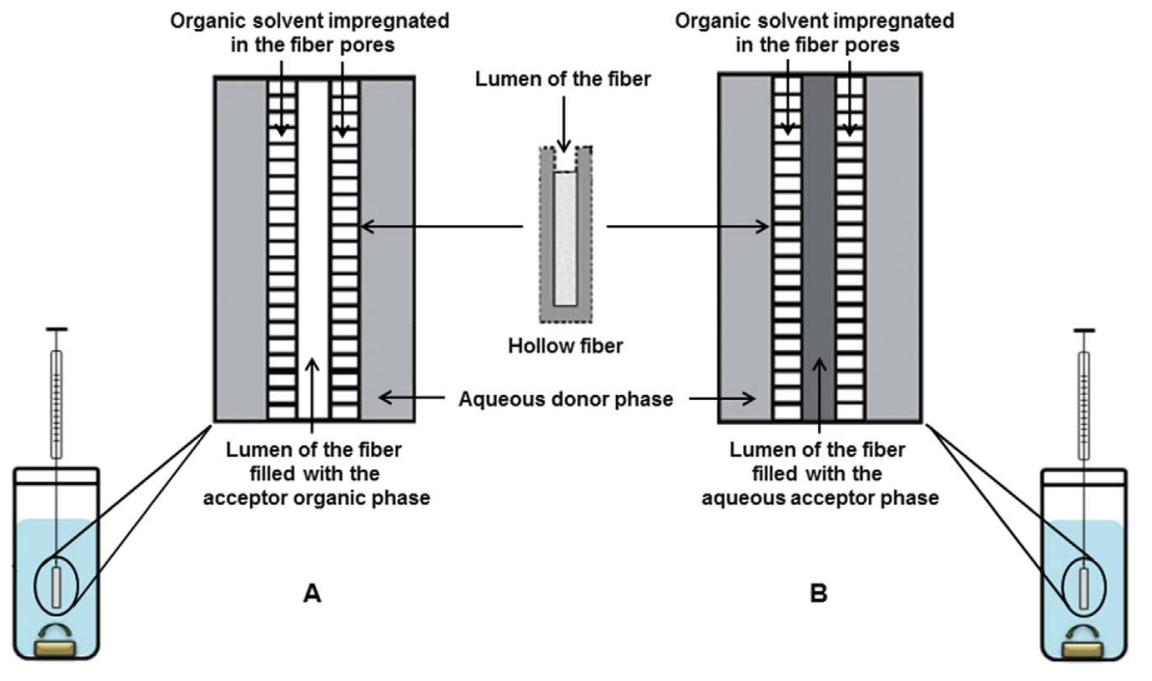

Fig. 7. Two-phase (A) and three-phase (B) systems involved in the HF-LPME technique.

HPLC-DAD. The applicability of the method was successfully confirmed by the extraction and determination of these drugs in milk, honey, fish and liver and muscles of lamb and chicken.

Based on the information presented in this section, one will find that the combination of HF-LPME with chromatographic systems proved successful for the extraction and detection of tetracyclines in food, biological and environmental samples. It should be noted that high enrichment factors, excellent sample clean-up within a short extraction time and low consumption of organic solvents are among the advantages of HF-LPME. In addition, the method allows the construction of different extraction units due to the high flexibility of the hollow fiber. Given the low cost of each extraction, the fiber can be used only once. This helps to avoid carry-over problems that is normally observed in other membrane extraction techniques $[85,133,135]$.

3.7.2.2. Dispersive liquid-liquid microextraction. Dispersive liquid-liquid microextraction (DLLME) is a novel and powerful preconcentration technique that uses a ternary solvent system to separate the analytes of interest from the sample matrix [140]. The technique involves the dispersion of an extraction solvent (water-immiscible) and a disperser solvent (miscible with water and the extraction solvent) in an aqueous solution; this results in a large area of contact between the aqueous phase and the extraction solvent. In this way, a rapid transfer of analytes from the aqueous phase to the extractive phase occurs due to the partitioning process. By so doing, the extraction process quickly reaches the equilibrium state and it is independent of time [93]. In conventional mode, an appropriate mixture of extraction and disperser solvents is rapidly injected into an aqueous sample containing the analytes of interest. The mixture is then gently shaken to form a cloudy solution with microdroplets. In doing so, the analytes in the aqueous sample are rapidly extracted into the fine droplets of the extraction solvent due to the increase in the contact surface between both phases (aqueous and organic). The extraction phase is separated from the cloudy mixture by centrifugation and the enriched analytes in the sedimented phase are determined by the most appropriate analytical technique [51,140-142]. Fig. 8 provides a simplified diagram of the steps involved in the DLLME technique.

Ultrasound-assisted dispersive liquid-liquid microextraction (USDLLME) was employed toward the development of a method for extracting tetracycline drugs from egg supplement samples [51]. Residues were rapidly determined by spectrophotometric FIA using a liquid waveguide capillary cell (LWCC) in-line with a controlled temperature heating bath. The samples were first treated with trichloroacetic acid to precipitate the proteins. Some factors affecting the extraction efficiency, such as the extraction solvent $(\mu \mathrm{L})$, disperser solvent $(\mu \mathrm{L})$, and sonication, extraction and centrifugation times, were optimized by

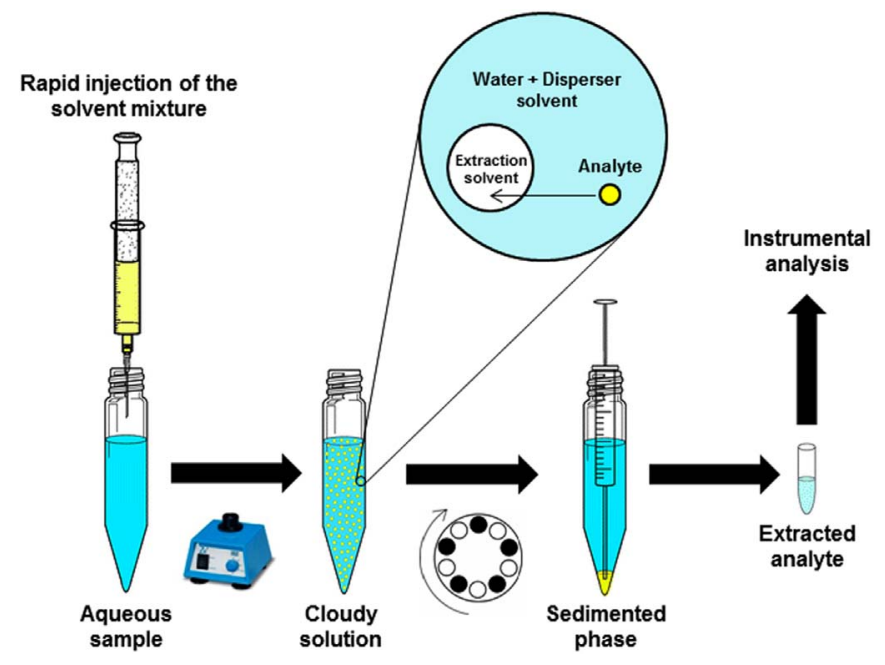

Fig. 8. Schematic diagram representing the steps involved in the DLLME technique.

multivariate analysis. Chloroform was found to be most effective for the extraction of individual tetracyclines. With regard to dispersive solvents, ethanol and acetonitrile exhibited the best overall recoveries probably due to better dispersion of the extraction solvent (chloroform) in the aqueous phase. Nevertheless, ethanol was chosen as the best dispersive solvent owing to the fact that it is a greener solvent and relatively less costly. It is worth pointing out that tetracycline recovery was reported to have improved by approximately $6 \%$ when the microextraction was assisted by ultrasound.

An obvious disadvantage of the traditional DLLME is that it requires the use of organic solvents such as extraction and dispersive solvents [143]. Ionic liquids (ILs) are organic salts consisting exclusively of organic cations paired with organic or inorganic anions. These liquids have been widely applied as possible "green solvents" in various fields of chemistry. The compounds often present melting points below or equal to $100{ }^{\circ} \mathrm{C}$. The greatest importance of ionic liquids lies in the possibility of designing their chemical structure in such a way to produce the desired properties, including elevated thermal stability, low vapor pressure, tunable viscosity, and miscibility with other solvents, as well as the capability of undergoing numerous solvation interactions. In general, ILs are non-toxic and non-flammable, as such, they are considered less polluting to the atmosphere and less dangerous for the analysts compared to traditional organic solvents. As a result, ionic liquid-based microextraction techniques have been the focus of study during the last few years. The core objective of the use of these 
techniques is to improve extraction and enrichment performance beyond those of the conventional sample preparation techniques $[125,144]$.

In this context, Song et al. developed an ionic liquid-based dispersive liquid-liquid microextraction (IL-DLLME) procedure combined with HPLC aimed at determining tetracycline drugs in eggs [145]. Among the four ILs tested, 1-butyl-3-methylimidazolium hexafluorophosphate showed the best extraction performance. To optimize the microextraction procedure, several parameters found to influence the extraction efficiency (ionic liquid and its volume, disperser solvent and its volume, extraction and centrifugation time, $\mathrm{pH}$ and salt addition) were investigated.

More recently, a novel naphthoic acid-based ionic liquid was developed and optimized as a non-organic solvent for the microextraction of tetracycline antibiotics from milk and chicken eggs, followed by HPLC-UV determination [66]. In that work, the synthesized ionic liquid 1-butyl-3-methylimidazolium naphthoic acid salt ([C4MIM] [NPA]) was used as extraction solvent while a conventional ionic liquid of 1-ethyl3-methylimidazolium tetrafluoroborate ([C2MIM] [BF4]) was employed as disperser solvent. The use of [C4MIM] [NPA] in functionalized ionic liquid-based NOSM method led to an increase in extraction efficiency by more than $20 \%$. It also modified the $\mathrm{pH}$ of the process because of its strong acidity, thus helping to rule out an additional $\mathrm{pH}$ adjustment step.

As with all other sample preparation techniques, there are certain constraints associated with DLLME. One of such limitations is the difficulty in automating the procedure by virtue of the need for phase separation steps. Moreover, the extracting solvent does not isolate the analytes from the soluble coextractives; this can cause errors in the quantification and affect the accuracy and precision of the results due to the matrix effect. The HF-LPME technique presents difficulty in analyzing highly polar and hydrophilic compounds. In view of that, it becomes necessary to add carrier agents in the sample that allow an increase in the permeability of these analytes in the hydrophobic membrane. Another current disadvantage of HF-LPME is the lack of commercially available equipment. The extraction units are usually cut to appropriate length and sealed using manual operation procedures, which affects the method precision. This shortcoming has imposed constraints on the application of HF-LPME in routine laboratories $[85,146]$.

Despite the demerits associated with these techniques, their useful benefits have rendered them suitable for application in the extraction and preconcentration of various types of analytes in different matrices. Their utilization has contributed towards achieving high preconcentration factors. In general, LPME is simple, affordable, rapid, selective, and easy to operate, apart from yielding high recovery of the analytes. Its importance lies in the considerable reduction or even elimination of the toxic solvents used [133]. In sample preparation procedures developed in recent years, these common solvents are often replaced by ILs, which are gaining widespread recognition as novel solvents in chemistry. The wider preference of ILs over common solvents is attributed to the fact that the former are considered less polluting to the atmosphere and less hazardous to the user. Many researchers regard ILs as possible green solvents [147,148]. The implementation of environmentally safer separation processes plays an increasingly important role in the development of extraction technology.

\section{Concluding remarks}

This work presents an overview of recent advances in sample preparation technologies for the determination of tetracycline residues in foodstuffs, focusing on milk and egg matrices. We have thoroughly reviewed the main extraction and clean-up techniques that are currently applied in the field. Most articles in the literature espouse the need for carrying out sample treatment before the cleaning step. Few methods were capable of detecting tetracycline residues in animal-derived foods without an appropriate sample treatment. This can be explained by the nature of complexity of food matrices.

Food analysis exhibits a clear trend toward the development of methods for determining a broad range of compounds in a single analysis step. Direct analysis in real time mass spectrometry (DART-MS) stands out among the discussed analytical techniques; this is by virtue of the fact that it allows the determination of both major and minor components in complex matrices with minimal or even no sample preparation. This rapid developing technique blended with the continuous advances in high resolution mass analyzers can fulfill the promise of a simple, rapid and high-throughput mass spectral analysis in situ of a large variety of samples. Nonetheless, from our investigation, we noted lack of scientific reports that shed light on the use of DARTMS schemes for tetracycline detection. While DART-MS is recognized as the best technique among the lots, one cannot ignore the fact that the lack of a separation step prior to ionization during the application of the technique can often result in very pronounced signal suppression and consequently lower detection selectivity. In this sense, sample treatment efficiency and mass resolution are key factors to consider when one chooses to perform analyses using DART-MS. Despite its limitations, DART-MS is regarded an emerging frontier technology for evaluating food authenticity and safety.

To date, sample preparation remains the crucial step for tetracycline drug determination in foodstuffs. The main efforts in this field have been directed toward the optimization of the preparation, extraction and clean-up steps, as well as the improvement of environmental compatibility of these procedures. Clearly, the development and application of new extraction approaches in the near future will undoubtedly help in routine monitoring, and will imply a drastic reduction of both work effort and time.

The isolation of tetracycline residues from food samples such as milk and egg is commonly aided by an organic solvent, usually acetonitrile or methanol, or by the combination of these solvents with acid compounds or buffer solutions. These extraction methods are often accompanied by cleaning steps for the separation of co-extracted matrix components aiming at obtaining a more purified sample extract for subsequent analysis using an appropriate technique.

Concerning the cleaning methods, SPE has become the most widely used approach for purification and preconcentration of these types of compounds in foods such as milk and eggs. This can be attributed to its simplicity, low cost, quickness, reduced time, low consumption of organic solvent, and availability of a wide range of sorbents. However, a major concern in the analysis of tetracyclines using traditional SPE sorbents lies in the tendency of the compounds to bind irreversibly to silanol groups contained in silica-based materials (C8, C18). These silanol groups either interact with the TC ketone groups or form chelate complexes with metal ions, which may result in low recovery yields during sample pre-treatment. Thus, these sorbents are prone to interferences by impurities in complex samples, and their cartridges are for single use only. Progress made in more efficient materials for SPE drives the trend towards more sensitive detection together with high sample throughput and less time devoted to sample clean-up.

Magnetically assisted chemical separation, which uses magnetite nano- or micron-sized particles coated with silica, has attracted great research interest in sample preparation over the last two decades. This technology has proved to be ideal for MSPE, in that silica shell presents reliable stability in aqueous media besides having a large surface area. The use of MSPE helps overcome the problem of column packing by simply using an external magnetic field.

Selective sorbents like MIPs are perhaps among the most innovative materials in the sample preparation field, even for the analysis of tetracyclines in foods. This molecular imprinting technology has drawn the attention of many researchers as an attractive alternative for the analysis of complex samples. The attractiveness of MIPs is attributed to their ability to selectively retain analytes and exclude macromolecules. 
MIP-based sorbents can be used several times. These sorbents offer a much more selective analyte extraction than conventional SPE sorbents. The main problem here has to do with the selectivity of the analyte in the presence of the matrix interferents. Rigidity, physical robustness, resistance to elevated temperatures and pressures, and inertness towards acids, bases, metal ions and organic solvents are among the advantages of MIPs. The main drawbacks of these more selective sorbents are that they are expensive and less suitable for harsh environments.

The current trend toward miniaturization of sample preparation procedures has resulted in the development of several microextraction techniques. These techniques are characterized by low cost, high extraction efficiency, reduced solvent amount and ease of device automation. Besides that, they also allow a relatively straightforward adaptation of the methods for on-site analysis. Miniaturization and integration allow the shortening of the sample extraction route. They are likewise highly effective in saving analysis time and in improving reproducibility compared with the manual protocols. Admittedly, the introduction of automated techniques usually leads to a rise in costs. As reported in the present review, dispersive-SPME, HF-LPME, and DLLME are miniaturized extraction techniques developed from traditional SPE or LLE. These techniques have gained popularity in the analysis of tetracycline residues in foods, such as milk and eggs. The success of microextraction lies in the improved analytical performance that can be achieved when this kind of techniques is applied. In addition to that, several properties pertinent to microextraction indicate that these miniaturized techniques are within the framework of green chemistry.

Regarding the new solvents applied in extraction and microextraction methods, recent advances have been embedded on the employment of solvents with low toxicity, such as ionic liquids. We expect to see further development of these new solvents because their unique properties bring great versatility and possible green character for application in various chemistry fields. Furthermore, ionic liquids are compatible with a variety of instrumental techniques. The study of ionic liquids has become an increasingly growing and promising field of research that is devoted to eliminating the use of dangerous chemical products. The methods involving the use of these new "green" solvents along with the traditional miniaturization procedure, undoubtedly, contribute toward the reduction of harmful impacts of analytical activities.

Overall, to date, the number of different extraction and clean-up techniques and their application toward the determination of tetracycline residues has seen an overwhelming growth. Fundamentally, these techniques are developed aiming at improving compatibility with modern analytical instruments in addition to contributing toward the avoidance of the use of hazardous chemicals and allowing sampling and pretreatment of the most varied food products. Despite the existence of valuable studies in this area, there still remains the quest for developing sample preparation procedures that can be widely applied for analysis of tetracycline antibiotics in foodstuffs. Such procedures should also be capable of exhibiting suitable operational features, including simplicity, quickness, miniaturization, automation, costlessness and safety. The implementation of environmentally safer separation processes plays an increasingly important role in the development of extraction technology.

\section{Acknowledgments}

The authors are sincerely grateful to National University of the Northeast (SGCyT-UNNE) and to National Scientific and Technical Research Council (CONICET) for the postdoctoral scholarship granted to M. Pérez-Rodríguez.

\section{Conflicts of interest}

The authors declare no conflicts of interest.

\section{References}

[1] M.P. Rodríguez, H.R. Pezza, L. Pezza, Simple and clean determination of tetracyclines by flow injection analysis, Spectrochim. Acta A 153 (2016) 386-392.

[2] J.A. Rodriguez, J. Espinosa, K. Aguilar-Arteaga, I.S. Ibarra, J.M. Miranda, Determination of tetracyclines in milk samples by magnetic solid phase extraction flow injection analysis, Microchim. Acta 171 (2010) 407-413.

[3] B.F. Spisso, A.L. de Oliveira e Jesus, M.A.G. de Araújo Júnior, M.A. Monteiro, Validation of a high-performance liquid chromatographic method with fluorescence detection for the simultaneous determination of tetracyclines residues in bovine milk, Anal. Chim. Acta 581 (2007) 108-117.

[4] A. Önal, Overview on liquid chromatographic analysis of tetracycline residues in food matrices, Food Chem. 127 (2011) 197-203.

[5] E.C. Pereira-Maia, P.P. Silva, W.B. De Almeida, H.F. Dos Santos, B.L. Marcial, R. Ruggiero, W. Guerra, Tetraciclinas e glicilciclinas: Uma visão geral, Quim. Nova 33 (2010) 700-706.

[6] M.M. Aguilera-Luiz, J.L.M. Vidal, R. Romero-González, A.G. Frenich, Multi-residue determination of veterinary drugs in milk by ultra-high-pressure liquid chromatography-tandem mass spectrometry, J. Chromatogr. A 1205 (2008) 10-16.

[7] A.V. Portugal, Sistemas de produção de alimentos de origem animal no futuro, Rev. Port. Ciênc. Vet. 97 (2000) 63-70.

[8] R.A. Mota, K.P. Chaves, D. Silva, M. Figueiroa, L. De Freitas, W. José, N. Porto, L.B. Galiza, Utilização indiscriminada de antimicrobianos e sua contribuição a multirresitência bacteriana, Braz. J. Vet. Res. Anim. Sci. 42 (2005) 465-470.

[9] C.K. Prado, M. Machinski Junior, Metodologia analítica para determinação de resíduos de tetraciclinas em leite: uma revisão, Rev. Inst. Adolfo Lutz 70 (2011) 448-456.

[10] S. Sczesny, H. Nau, G. Hamscher, Residue analysis of tetracyclines and their metabolites in eggs and in the environment by HPLC coupled with a microbiological assay and tandem mass spectrometry, J. Agric. Food Chem. 51 (2003) 697-703.

[11] M. Piatkowska, P. Jedziniak, J. Zmudzki, Comparison of different sample preparation procedures for multiclass determination of selected veterinary drug, coccidiostat and insecticide residues in eggs by liquid chromatography-tandem mass spectrometry, Anal. Methods 6 (2014) 3034-3044.

[12] T.A. McGlinchey, P.A. Rafter, F. Regan, G.P. McMahon, A review of analytical methods for the determination of aminoglycoside and macrolide residues in food matrices, Anal. Chim. Acta 624 (2008) 1-15.

[13] Rome Declaration on World Food Security and World Food Summit Plan of Action, in: WORLD FOOD SUMMIT, Rome, 1996.

[14] J. Caswell, Valuing the benefits and costs of improved food safety and nutrition, Aust. J. Agric. Resour. Econ. 42 (1998) 409-424.

[15] M.D. Marazuela, S. Bogialli, A review of novel strategies of sample preparation for the determination of antibacterial residues in foodstuffs using liquid chromatography-based analytical methods, Anal. Chim. Acta 645 (2009) 5-17.

[16] Codex Alimentarius, in: International Food Standards, n.d. 〈http://www.fao.org/ fao-who-codexalimentarius/en/ $>$.

[17] Codex Alimentarius, CAC/MRL-2, Maximum residues limits (MRLs) and risk management recommendations (RMRs) for residues of veterinary drugs in Foods, 8, 2015.

[18] European Union (EU), Commission regulation no. 37/2010, On pharmacologically active substances and their classification regarding maximum residue limits in foodstuffs of animal origin, Off. J. Eur. Union L15 (2010) 19-64.

[19] Canada, Food and Drugs Act SOR-87, List of maximum residue limits (MRLs) for veterinary drugs in foods, Can. Gaz. Part II 147 (2013) 3-21.

[20] China, Ministry of Agriculture of the People's Republic of China, Regulation No. $235 / 2002$, Concerning maximum residue limits of veterinary drugs in foodstuffs of animal origin.

[21] Brazil, Plano Nacional de Controle de Resíduos e Contaminantes (PNCRC), MAPA Instrução Normativa SDA No. 11, 7, 2014.

[22] Sociedade Brasileira de Alimentação e Nutrição (SBAN), A importância do consumo de leite no atual cenário nutricional brasileiro, São Paulo, 1-27, 2015.

[23] A.G. Frenich, M. del M. Aguilera-Luiz, J.L.M. Vidal, R. Romero-González, Comparison of several extraction techniques for multiclass analysis of veterinary drugs in eggs using ultra-high pressure liquid chromatography-tandem mass spectrometry, Anal. Chim. Acta 661 (2010) 150-160.

[24] F.M.F. Cunha, M.B.H. Magalhães, D.S. Bonnas, Desafios da gestão da segurança dos alimentos em unidades de alimentação e nutrição no Brasil: uma revisão, Rev. Comport. Cult. Soc. 1 (2013) 4-14.

[25] AOAC Official Method 995.04, Multiple tetracycline residues in milk, Official Methods of Analysis of AOAC INTERNATIONAL, 19th ed., 2012.

[26] AOAC Official Method 995.09, Chlortetracycline, Oxytetracycline, and Tetracycline in Edible Animal Tissues, Official Methods of Analysis of AOAC INTERNATIONAL, 19th ed., 2012.

[27] G.J. Palm, T. Lederer, P. Orth, W. Saenger, M. Takahashi, W. Hillen, W. Hinrichs, Specific binding of divalent metal ions to tetracycline and to the Tet repressor/ tetracycline complex, J. Biol. Inorg. Chem. 13 (2008) 1097-1110.

[28] M. Denobile, E. de, S. Nascimento, Validação de método para determinação de resíduos dos antibióticos oxitetraciclina, tetraciclina, clortetraciclina e doxiciclina, em leite, por cromatografia líquida de alta eficiência, Rev. Bras. Ciênc. Farm. 40 (2004) 209-218.

[29] C. Esteban, C.A. Rodrigues, E.D.S. Nascimento, Determinação de tetraciclina em líquido sinovial de vacas com doença podal, Rev. Bras. Ciênc. Farm. 43 (2007) 205-214.

[30] H. Oka, Y. Ito, H. Matsumoto, Chromatographic analysis of tetracycline antibiotics in foods, J. Chromatogr. A 882 (2000) 109-133. 
[31] R. Zanella, O.D. Prestes, A. Friggi, M.L. Martins, M.B. Adaime, An overview about recent advances in sample preparation techniques for pesticide residues analysis in cereals and feedstuffs, Pestic. - Recent Trends Pestic. Residue Assay (2012) 149-170.

[32] J. Hajslova, T. Cajka, L. Vaclavik, Challenging applications offered by direct analysis in real time (DART) in food-quality and safety analysis, Trends Anal. Chem. 30 (2011) 204-218.

[33] D. Yi-yang, L. Jia-hui, W. Sai, C. Qi-long, G. Tian-yang, Z. Li-ya, J. Yong, S. Hai-jia, T. Tian-wei, Emerging frontier technologies for food safety analysis and risk assessment, J. Integr. Agric. 14 (2015) 2231-2242.

[34] J.H. Gross, Direct analysis in real time - a critical review on DART-MS, Anal. Bioanal. Chem. 406 (2013) 63-80.

[35] L. Li, H. Hsieh, C. Hsu, Clinical application of ambient ionization mass spectrometry, Mass. Spectrom. 6 (2017) S0060.

[36] L. Vaclavik, T. Cajka, V. Hrbek, J. Hajslova, Ambient mass spectrometry employing direct analysis in real time (DART) ion source for olive oil quality and authenticity assessment, Anal. Chim. Acta 645 (2009) 56-63.

[37] S.E. Edison, L.A. Lin, B.M. Gamble, J. Wong, K. Zhang, Surface swabbing technique for the rapid screening for pesticides using ambient pressure desorption ionization with high-resolution mass spectrometry, Rapid Commun. Mass Spectrom. 25 (2011) 127-139.

[38] F.J. Lara, D. Chan, M. Dickinson, A.S. Lloyd, S.J. Adams, Evaluation of direct analysis in real time for the determination of highly polar pesticides in lettuce and celery using modified quick polar pesticides extraction method, J. Chromatogr. A 1496 (2017) 37-44.

[39] T. Cajka, K. Riddellova, M. Tomaniova, J. Hajslova, Ambient mass spectrometry employing a DART ion source for metabolomic fingerprinting/profiling: a powerful tool for beer origin recognition, Metabolomics 7 (2011) 500-508.

[40] A.D. Lesiak, R.B. Cody, A.J. Dane, R.A. Musah, Plant seed species identification from chemical fingerprints: a high-throughput application of direct analysis in real time mass spectrometry, Anal. Chem. 87 (2015) 8748-8757.

[41] J. Srbek, B. Klejdus, M. Douša, J. Břicháč, P. Stasiak, J. Reitmajer, L. Nováková, Direct analysis in real time - high resolution mass spectrometry as a valuable tool for the pharmaceutical drug development, Talanta 130 (2014) 518-526.

[42] A.D. Lesiak, R.B. Cody, M. Ubukata, R.A. Musah, Direct analysis in real time high resolution mass spectrometry as a tool for rapid characterization of mind-altering plant materials and revelation of supplement adulteration - the case of Kanna, Forensic Sci. Int. 260 (2016) 66-73.

[43] J. Wang, J. Zhu, L. Si, Q. Du, H. Li, W. Bi, D.D.Y. Chen, High throughput screening of phenoxy carboxylic acids with dispersive solid phase extraction followed by direct analysis in real time mass spectrometry, Anal. Chim. Acta 996 (2017) 20-28.

[44] L. Vaclavik, J. Rosmus, B. Popping, J. Hajslova, Rapid determination of melamine and cyanuric acid in milk powder using direct analysis in real time-time-of-flight mass spectrometry, J. Chromatogr. A 1217 (2010) 4204-4211.

[45] E.S. Chernetsova, G.E. Morlock, Assessing the capabilities of direct analysis in real time mass spectrometry for 5-hydroxymethylfurfural quantitation in honey, Int. J. Mass Spectrom. 314 (2012) 22-32.

[46] M.P. Rodríguez, B. Ferreira da Silva, H.R. Pezza, L. Pezza, A greener flow injection method based on a LWCC for the screening of tetracycline antibiotics in bovine milk samples, Anal. Methods 8 (2016) 5262-5271.

[47] K. Ridgway, S.P.D. Lalljie, R.M. Smith, Sample preparation techniques for the determination of trace residues and contaminants in foods, J. Chromatogr. A 1153 (2007) 36-53.

[48] S.M. Taghdisi, N.M. Danesh, M. Ramezani, K. Abnous, A novel M-shape electrochemical aptasensor for ultrasensitive detection of tetracyclines, Biosens. Bioelectron. 85 (2016) 509-514.

[49] J. Zhang, B. Zhang, Y. Wu, S. Jia, T. Fan, Z. Zhang, C. Zhang, Fast determination of the tetracyclines in milk samples by the aptamer biosensor, Analyst 135 (2010) 2706-2710.

[50] L.Q. Wang, F.Y. Lin, L.P. Yu, A molecularly imprinted photonic polymer sensor with high selectivity for tetracyclines analysis in food, Analyst 137 (2012) 3502-3509.

[51] M.P. Rodríguez, H.R. Pezza, L. Pezza, Ultrasound-assisted dispersive liquid-liquid microextraction of tetracycline drugs from egg supplements before flow injection analysis coupled to a liquid waveguide capillary cell, Anal. Bioanal. Chem. 408 (2016) 6201-6211.

[52] K. Iwaki, N. Okumura, M. Yamazaki, Determination of tetracycline antibiotics by reversed-phase high-performance liquid chromatography with fluorescence detection, J. Chromatogr. A 623 (1992) 153-158.

[53] M.C.V. Mamani, F.G.R. Reyes, S. Rath, Multiresidue determination of tetracyclines, sulphonamides and chloramphenicol in bovine milk using HPLC-DAD, Food Chem. 117 (2009) 545-552.

[54] M.E. Dasenaki, N.S. Thomaidis, Multi-residue determination of 115 veterinary drugs and pharmaceutical residues in milk powder, butter, fish tissue and eggs using liquid chromatography-tandem mass spectrometry, Anal. Chim. Acta 880 (2015) 103-121.

[55] Y. Liu, H. Yang, S. Yang, Q. Hu, H. Cheng, H. Liu, Y. Qiu, High-performance liquid chromatography using pressurized liquid extraction for the determination of seven tetracyclines in egg, fish and shrimp, J. Chromatogr. B 917-918 (2013) 11-17.

[56] H. Filik, A A Avan, S. Aydar, D. Ozyurt, B. Demirata, Determination of tetracycline on the surface of a high-performance graphene modified screen-printed carbon electrode in milk and honey samples, Curr. Nanosci. 12 (2016) 527-533.

[57] H. De Ruyck, H. De Ridder, Determination of tetracycline antibiotics in cow's milk by liquid chromatography/tandem mass spectrometry, Rapid Commun. Mass Spectrom. 21 (2007) 1511-1520.

[58] L. Vera-Candioti, A.C. Olivieri, H.C. Goicoechea, Development of a novel strategy for preconcentration of antibiotic residues in milk and their quantitation by capillary electrophoresis, Talanta 82 (2010) 213-221.

[59] S.M. Santos, M. Henriques, A.C. Duarte, V.I. Esteves, Development and application of a capillary electrophoresis based method for the simultaneous screening of six antibiotics in spiked milk samples, Talanta 71 (2007) 731-737.

[60] N. Rodríguez, B.D. Real, M.C. Ortiz, L.A. Sarabia, A. Herrero, Usefulness of parallel factor analysis to handle the matrix effect in the fluorescence determination of tetracycline in whey milk, Anal. Chim. Acta 632 (2009) 42-51.

[61] Q. Wang, Z. Lv, Q. Tang, C. Bin Gong, M.H.W. Lam, X.B. Ma, C.F. Chow, Photoresponsive molecularly imprinted hydrogel casting membrane for the determination of trace tetracycline in milk, J. Mol. Recognit. 29 (2016) 123-130.

[62] M.X. Feng, G.N. Wang, K. Yang, H.Z. Liu, J.P. Wang, Molecularly imprinted polymer-high performance liquid chromatography for the determination of tetracycline drugs in animal derived foods, Food Control 69 (2016) 171-176.

[63] K.I. Nikolaidou, V.F. Samanidou, I.N. Papadoyannis, Development and validation of an HPLC multi-residue method for the determination of seven tetracycline antibiotic residues in bovine liver and kidney according to the European union decision 2002/657/EC, J. Liq. Chromatogr. Relat. Technol. 31 (2008) 2523-2540.

[64] M.C. Carson, M.A. Ngoh, S.W. Hadley, Confirmation of multiple tetracycline residues in milk and oxytetracycline in shrimp by liquid chromatography-particle beam mass spectrometry, J. Chromatogr. B 712 (1998) 113-128.

[65] J. Xue, J. Liu, C. Wang, Y. Tian, N. Zhou, Simultaneous electrochemical detection of multiple antibiotic residues in milk based on aptamers and quantum dots, Anal. Methods 8 (2016) 1981-1988.

[66] J. Gao, H. Wang, J. Qu, H. Wang, X. Wang, Development and optimization of a naphthoic acid-based ionic liquid as a "non-organic solvent microextraction" for the determination of tetracycline antibiotics in milk and chicken eggs, Food Chem. 215 (2017) 138-148.

[67] W.C. Andersen, J.E. Roybal, S.A. Gonzales, S.B. Turnipseed, A.P. Pfenning, L.R. Kuck, Determination of tetracycline residues in shrimp and whole milk using liquid chromatography with ultraviolet detection and residue confirmation by mass spectrometry, Anal. Chim. Acta 529 (2005) 145-150.

[68] V. Jiménez, A. Rubies, F. Centrich, R. Companyó, J. Guiteras, Development and validation of a multiclass method for the analysis of antibiotic residues in eggs by liquid chromatography-tandem mass spectrometry, J. Chromatogr. A 1218 (2011) 1443-1451.

[69] G. Mu, H. Liu, L. Xu, L. Tian, F. Luan, Matrix solid-phase dispersion extraction and capillary electrophoresis determination of tetracycline residues in milk, Food Anal. Methods 5 (2012) 148-153.

[70] M. Piatkowska, P. Jedziniak, J. Zmudzki, Multiresidue method for the simultaneous determination of veterinary medicinal products, feed additives and illegal dyes in eggs using liquid chromatography-tandem mass spectrometry, Food Chem. 197 (2016) 571-580.

[71] X. Sun, X. He, Y. Zhang, L. Chen, Determination of tetracyclines in food samples by molecularly imprinted monolithic column coupling with high performance liquid chromatography, Talanta 79 (2009) 926-934.

[72] X.F. Jia, B. Shao, Y.N. Wu, Y. Yang, J. Zhang, Simultaneous determination of tetracylines and quinolones antibiotics in egg by ultra-performance liquid chromatography-electrospray tandem mass spectrometry, J. AOAC Int. 91 (2008) 461-468.

[73] S. Wang, P. Yang, Y. Cheng, Analysis of tetracycline residues in bovine milk by CEMS with field-amplified sample stacking, Electrophoresis 28 (2007) 4173-4179.

[74] P. Masawat, S. Mekprayoon, S. Liawruangrath, S. Upalee, N. Youngvises, On-line preconcentration and determination of tetracycline residues in milk using solidphase extraction in conjunction with flow injection spectrophotometry, Maejo Int. J. Sci. Technol. 2 (2008) 418-430.

[75] M.M. Abbasi, H. Babaei, M. Ansarin, A.O.S. Nourdadgar, M. Nemati, Simultaneous determination of tetracyclines residues in bovine milk samples by solid phase extraction and HPLC-FL method, Adv. Pharm. Bull. 1 (2011) 34-39.

[76] X.Q. Yang, C.X. Yang, X.P. Yan, Zeolite imidazolate framework-8 as sorbent for on line solid-phase extraction coupled with high-performance liquid chromatography for the determination of tetracyclines in water and milk samples, J. Chromatogr. A 1304 (2013) 28-33.

[77] Y.K. Lv, J.Q. Zhang, Z.Y. Guo, W. Zhang, H.W. Sun, Determination of tetracyclines residues in egg, milk, and milk powder by online coupling of a precolumn packed with molecular imprinted hybrid composite materials to RP-HPLC-UV, J. Liq. Chromatogr. Relat. Technol. 38 (2015) 1-7.

[78] J. Li, L. Chen, X. Wang, H. Jin, L. Ding, K. Zhang, H. Zhang, Determination of tetracyclines residues in honey by on-line solid-phase extraction high-performance liquid chromatography, Talanta 75 (2008) 1245-1252.

[79] A.A. Yakout, D.A. El-Hady, A combination of $\beta$-cyclodextrin functionalized magnetic graphene oxide nanoparticles with $\beta$-cyclodextrin-based sensor for highly sensitive and selective voltammetric determination of tetracycline and doxycycline in milk samples, RSC Adv. 6 (2016) 41675-41686.

[80] D.N. Heller, C.B. Nochetto, N.G. Rummel, M.H. Thomas, Development of multiclass methods for drug residues in eggs: hydrophilic solid-phase extraction cleanup and liquid chromatography/tandem mass spectrometry analysis of tetracycline, fluoroquinolone, sulfonamide, and $\beta$-lactam residues, J. Agric. Food Chem. 54 (2006) 5267-5278.

[81] Z. Zhang, X. Li, S. Ding, H. Jiang, J. Shen, X. Xia, Multiresidue analysis of sulfonamides, quinolones, and tetracyclines in animal tissues by ultra-high performance liquid chromatography-tandem mass spectrometry, Food Chem. 204 (2016) 252-262.

[82] E. Thessalonikeos, H. Tsoukali, K. Spagou, M. Vlachou, A. Pouliopoulos, N. Raikos, Development of a liquid-liquid extraction procedure for the analysis of amphetamine in biological specimens by GC-FID, Open Forensic Sci. J. 2 (2009) 12-15.

[83] V. Pichon, Solid-phase extraction for multiresidue analysis of organic 
contaminants in water, J. Chromatogr. A 885 (2000) 195-215.

84] A. Galuszka, Z. Migaszewski, J. Namiesnik, The 12 principles of green analytical chemistry and the SIGNIFICANCE mnemonic of green analytical practices, Trends Anal. Chem. 50 (2013) 78-84.

[85] E.H.C. Morais, F.R. Begnini, I.C.S.F. Jardim, Técnicas de preparo de amostra empregadas na determinação de agrotóxicos carbamatos em água e solo, Sci. Chromatogr. 5 (2013) 146-162.

[86] E. Karageorgou, M. Armeni, I. Moschou, V. Samanidou, Ultrasound-assisted dispersive extraction for the high pressure liquid chromatographic determination of tetracyclines residues in milk with diode array detection, Food Chem. 150 (2014) 328-334.

[87] M. Anastassiades, S.J. Lehotay, D. Stajnbaher, F.J. Schenck, Fast and easy multi residue method employing acetonitile extraction/partitioning and "dispersive solid-phase extraction" for the determination of pesticide residues in produce, $\mathrm{J}$. AOCA Int. 86 (2003) 412-431.

[88] O.D. Prestes, M.B. Adaime, R. Zanella, QuEChERS: possibilidades e tendências no preparo de amostra para determinação multirresíduo de pesticidas em alimentos, Sci. Chromatogr. 3 (2011) 51-64.

[89] O.D. Prestes, C.A. Friggi, M.B. Adaime, R. Zanella, QuEChERS - Um método moderno de preparo de amostra para determinação multirresíduo de pesticidas em alimentos por métodos cromatográficos acoplados à espectrometria de massas, Quim. Nova 32 (2009) 1620-1634.

[90] A.L. Capriotti, C. Cavaliere, S. Piovesana, R. Samperi, A. Laganà, Multiclass screening method based on solvent extraction and liquid chromatography-tandem mass spectrometry for the determination of antimicrobials and mycotoxins in egg, J. Chromatogr. A 1268 (2012) 84-90.

[91] W.H. Tsai, T.C. Huang, J.J. Huang, Y.H. Hsue, H.Y. Chuang, Dispersive solid-phase microextraction method for sample extraction in the analysis of four tetracyclines in water and milk samples by high-performance liquid chromatography with diode-array detection, J. Chromatogr. A 1216 (2009) 2263-2269.

[92] A. Andrade-Eiroa, M. Canle, V. Leroy-Cancellieri, V. Cerda, Solid-phase extraction of organic compounds: a critical review (Part I), Trends Anal. Chem. 80 (2016) 655-667.

[93] M.L. Martins, E.G. Primel, S.S. Caldas, O.D. Prestes, M.B. Adaime, R. Zanella, Microextração Líquido-Líquido Dispersiva (DLLME): fundamentos e aplicações, Sci. Chromatogr. 4 (2012) 35-51.

[94] A. Andrade-Eiroa, M. Canle, V. Leroy-Cancellieri, V. Cerdà, Solid-phase extraction of organic compounds: a critical review. Part ii, Trends Anal. Chem. 80 (2016) 655-667.

[95] F. Bruno, R. Curini, A. Di Corcia, M. Nazzari, M. Pallagrosi, An original approach to determining traces of tetracycline antibiotics in milk and eggs by solid-phase extraction and liquid chromatography/mass spectrometry, Rapid Commun. Mass Spectrom. 16 (2002) 1365-1376.

[96] W. Thanasarakhan, S. Kruanetr, R.L. Deming, B. Liawruangrath, S. Wangkarn, S. Liawruangrath, Sequential injection spectrophotometric determination of tetracycline antibiotics in pharmaceutical preparations and their residues in honey and milk samples using yttrium (III) and cationic surfactant, Talanta 84 (2011) 1401-1409.

[97] Q. Zhou, Y. Ding, J. Xiao, Simultaneous determination of cyanazine, chlorotoluron and chlorbenzuron in environmental water samples with SPE multiwalled carbon nanotubes and LC, Chromatographia 65 (2006) 25-30.

[98] J.J. Xu, M. An, R. Yang, Z. Tan, J. Hao, J. Cao, L.Q. Peng, W. Cao, Determination of tetracycline antibiotic residues in honey and milk by miniaturized solid phase extraction using Chitosan-modified graphitized multiwalled carbon nanotubes, J. Agric. Food Chem. 64 (2016) 2647-2654.

[99] T. Jing, X.D. Gao, P. Wang, Y. Wang, Y.F. Lin, X.Z. Hu, Q.L. Hao, Y.K. Zhou, S.R. Mei, Determination of trace tetracycline antibiotics in foodstuffs by liquid chromatography-tandem mass spectrometry coupled with selective molecularimprinted solid-phase extraction, Anal. Bioanal. Chem. 393 (2009) 2009-2018.

[100] T. Jing, J. Niu, H. Xia, Q. Dai, H. Zheng, Q. Hao, S. Mei, Y. Zhou, Online coupling of molecularly imprinted solid-phase extraction to HPLC for determination of trace tetracycline antibiotic residues in egg samples, J. Sep. Sci. 34 (2011) 1469-1476.

[101] L. Chen, J. Liu, Q. Zeng, H. Wang, A. Yu, H. Zhang, L. Ding, Preparation of magnetic molecularly imprinted polymer for the separation of tetracycline antibiotics from egg and tissue samples, J. Chromatogr. A 1216 (2009) 3710-3719.

[102] I.S. Ibarra, J.A. Rodriguez, J.M. Miranda, M. Vega, E. Barrado, Magnetic solid phase extraction based on phenyl silica adsorbent for the determination of tetracyclines in milk samples by capillary electrophoresis, J. Chromatogr. A 1218 (2011) 2196-2202.

[103] J.L. Urraca, M. Castellari, C.A. Barrios, M.C. Moreno-Bondi, Multiresidue analysis of fluoroquinolone antimicrobials in chicken meat by molecularly imprinted solidphase extraction and high performance liquid chromatography, J. Chromatogr. A 1343 (2014) 1-9.

[104] L. Chen, X. Wang, W. Lu, X. Wu, J. Li, Molecular imprinting: perspectives and applications, Chem. Soc. Rev. 45 (2016) 2137-2211.

[105] Y. Xiong, H. Zhou, Z. Zhang, D. He, C. He, Molecularly imprinted on-line solid phase extraction combined with flow-injection chemiluminescence for the determination of tetracycline, Analyst 131 (2006) 829-834.

[106] X. Kan, Q. Zhao, Z. Zhang, Z. Wang, J.J. Zhu, Molecularly imprinted polymers microsphere prepared by precipitation polymerization for hydroquinone recognition, Talanta 75 (2008) 22-26.

[107] V. Pichon, Selective sample treatment using molecularly imprinted polymers, J. Chromatogr. A 1152 (2007) 41-53.

[108] Y. Nakamura, S. Masumoto, H. Matsunaga, J. Haginaka, Molecularly imprinted polymer for glutathione by modified precipitation polymerization and its application to determination of glutathione in supplements, J. Pharm. Biomed. Anal.
144 (2017) 230-235.

[109] Q. Xia, Y. Yun, Q. Li, Z. Huang, Z. Liang, Preparation and characterization of monodisperse molecularly imprinted polymer microspheres by precipitation polymerization for kaempferol, Des. Monomers Polym. 20 (2017) 201-209.

[110] Y. Xiao, R. Xiao, J. Tang, Q. Zhu, X. Li, Y. Xiong, X. Wu, Preparation and adsorption properties of molecularly imprinted polymer via RAFT precipitation polymerization for selective removal of aristolochic acid I, Talanta 162 (2017) 415-422.

[111] X. Shen, C. Huang, S. Shinde, M. Switnicka-Plak, P.A.G. Cormack, B. Sellergren, Reflux precipitation polymerization: a new synthetic insight in molecular imprinting at high temperature, RSC Adv. 6 (2016) 81491-81499.

[112] G. Cheng, M. He, H. Peng, B. Hu, Dithizone modified magnetic nanoparticles for fast and selective solid phase extraction of trace elements in environmental and biological samples prior to their determination by ICP-OES, Talanta 88 (2012) 507-515.

[113] Y. Yu, Z. Fan, Determination of tetracyclines in bovine milk using laccaic acidloaded magnetite nanocomposite for magnetic solid-phase extraction, J. Chromatogr. Sci. (2017) 1-7.

[114] G. Zurhelle, E. Müller-Seitz, M. Petz, Automated residue analysis of tetracyclines and their metabolites in whole egg, egg white, egg yolk and hen's plasma utilizing a modified ASTED system, J. Chromatogr. B Biomed. Sci. Appl. 739 (2000) 191-203.

[115] A.L. Capriotti, C. Cavaliere, P. Giansanti, R. Gubbiotti, R. Samperi, A. Laganà, Recent developments in matrix solid-phase dispersion extraction, J. Chromatogr. A 1217 (2010) 2521-2532.

[116] S.A. Barker, Matrix solid phase dispersion (MSPD), J. Biochem. Biophys. Methods 70 (2007) 151-162.

[117] E.M. Kristenson, U.A.T. Brinkman, L. Ramos, Recent advances in matrix solidphase dispersion, TrAC - Trends Anal. Chem. 25 (2006) 96-111.

[118] V. Andreu, P. Vazquez-Roig, C. Blasco, Y. Picó, Determination of tetracycline residues in soil by pressurized liquid extraction and liquid chromatography tandem mass spectrometry, Anal. Bioanal. Chem. 394 (2009) 1329-1339.

[119] R.M. Alonso-Salces, E. Korta, A. Barranco, L.A. Berrueta, B. Gallo, F. Vicente, Pressurized liquid extraction for the determination of polyphenols in apple, J. Chromatogr. A 933 (2001) 37-43.

[120] A. Mustafa, C. Turner, Pressurized liquid extraction as a green approach in food and herbal plants extraction: a review, Anal. Chim. Acta 703 (2011) 8-18.

[121] K. Stoob, H.P. Singer, S. Stettler, N. Hartmann, S.R. Mueller, C.H. Stamm, Exhaustive extraction of sulfonamide antibiotics from aged agricultural soils using pressurized liquid extraction, J. Chromatogr. A 1128 (2006) 1-9.

[122] M. Valcárcel, S. Cárdenas, R. Lucena, Microextraction techniques, Anal. Bioanal. Chem. 406 (2014) 1999-2000.

[123] F. Augusto, E. Carasek, R.G.C. Silva, S.R. Rivellino, A.D. Batista, E. Martendal, New sorbents for extraction and microextraction techniques, J. Chromatogr. A 1217 (2010) 2533-2542.

[124] A.K. Malik, V. Kaur, N. Verma, A review on solid phase microextraction-high performance liquid chromatography as a novel tool for the analysis of toxic metal ions, Talanta 68 (2006) 842-849.

[125] T.D. Ho, A.J. Canestraro, J.L. Anderson, Ionic liquids in solid-phase microextraction: a review, Anal. Chim. Acta 695 (2011) 18-43.

[126] Q.H. Zhang, L. Di Zhou, H. Chen, C.Z. Wang, Z.N. Xia, C.S. Yuan, Solid-phase microextraction technology for in vitro and in vivo metabolite analysis, Trends Anal. Chem. 80 (2016) 57-65.

[127] W.H. Tsai, H.Y. Chuang, H.H. Chen, J.J. Huang, H.C. Chen, S.H. Cheng, T.C. Huang, Application of dispersive liquid-liquid microextraction and dispersive micro-solid-phase extraction for the determination of quinolones in swine muscle by high-performance liquid chromatography with diode-array detection, Anal. Chim. Acta 656 (2009) 56-62.

[128] F.J. Liu, C.T. Liu, W. Li, A.N. Tang, Dispersive solid-phase microextraction and capillary electrophoresis separation of food colorants in beverages using diamino moiety functionalized silica nanoparticles as both extractant and pseudostationary phase, Talanta 132 (2015) 366-372.

[129] N. Fontanals, R.M. Marcé, F. Borrull, New materials in sorptive extraction techniques for polar compounds, J. Chromatogr. A 1152 (2007) 14-31.

[130] A. Asfaram, M. Ghaedi, A. Goudarzi, M. Soylak, Comparison between dispersive liquid-liquid microextraction and ultrasound-assisted nanoparticles-dispersive solid-phase microextraction combined with microvolume spectrophotometry method for the determination of Auramine-O in water samples, RSC Adv. 5 (2015) 39084-39096.

[131] F. Pena-Pereira, I. Lavilla, C. Bendicho, Liquid-phase microextraction techniques within the framework of green chemistry, Trends Anal. Chem. 29 (2010) 617-628.

[132] H. Kataoka, Recent developments and applications of microextraction techniques in drug analysis, Anal. Bioanal. Chem. 396 (2010) 339-364.

[133] D. Han, K.H. Row, Trends in liquid-phase microextraction, and its application to environmental and biological samples, Microchim. Acta 176 (2012) 1-22.

[134] J.A. Ocaña-González, R. Fernández-Torres, M.A. Bello-López, M. Ramos-Payán, New developments in microextraction techniques in bioanalysis. A review, Anal. Chim. Acta 905 (2016) 8-23.

[135] V. Sharifi, A. Abbasi, A. Nosrati, Application of hollow fiber liquid phase microextraction and dispersive liquid-liquid microextraction techniques in analytical toxicology, J. Food Drug Anal. 24 (2016) 264-276.

[136] S.C.N. Queiroz, C.H. Collins, I.C.S.F. Jardim, Métodos de extração e/ou concentração de compostos encontrados em fluidos biológicos para posterior determinação cromatográfica, Quim. Nova 24 (2001) 68-76.

[137] F. Ghamari, A. Bahrami, Y. Yamini, F.G. Shahna, Development of hollow-fiber liquid-phase microextraction method for determination of urinary trans, transmuconic acid as a biomarker of benzene exposure, Anal. Chem. Insights 11 (2016) 
65-71.

[138] F. Tajabadi, M. Ghambarian, Y. Yamini, N. Yazdanfar, Combination of hollow fiber liquid phase microextraction followed by HPLC-DAD and multivariate curve resolution to determine antibacterial residues in foods of animal origin, Talanta 160 (2016) 400-409.

[139] S. Shariati, Y. Yamini, A. Esrafili, Carrier mediated hollow fiber liquid phase microextraction combined with HPLC-UV for preconcentration and determination of some tetracycline antibiotics, J. Chromatogr. B Anal. Technol. Biomed. Life Sci. 877 (2009) 393-400.

[140] M. Saraji, M.K. Boroujeni, Recent developments in dispersive liquid-liquid microextraction, Anal. Bioanal. Chem. 406 (2014) 2027-2066.

[141] M. Saraji, M. Marzban, Determination of 11 priority pollutant phenols in wastewater using dispersive liquid-liquid microextraction followed by high-performance liquid chromatography-diode-array detection, Anal. Bioanal. Chem. 396 (2010) 2685-2693.

[142] M.M. Parrilla Vázquez, P. Parrilla Vázquez, M. Martínez Galera, M.D. Gil García, A. Uclés, Ultrasound-assisted ionic liquid dispersive liquid-liquid microextraction coupled with liquid chromatography-quadrupole-linear ion trap-mass spectrometry for simultaneous analysis of pharmaceuticals in wastewaters, J. Chromatogr. A 1291 (2013) 19-26.

[143] M. Rajabi, S. Asemipour, B. Barfi, M.R. Jamali, M. Behzad, Ultrasound-assisted ionic liquid based dispersive liquid-liquid microextraction and flame atomic absorption spectrometry of cobalt, copper, and zinc in environmental water samples, J. Mol. Liq. 194 (2014) 166-171.

[144] J.-N. Sun, J. Chen, Y.-P. Shi, Multiple functional ionic liquids based dispersive liquid-liquid microextraction combined with high performance chromatography for the determination of phenolic compounds in water samples, Talanta 125 (2014) 329-335.

[145] J. Song, Z.H. Zhang, Y.Q. Zhang, C. Feng, G.N. Wang, J.P. Wang, Ionic liquid dispersive liquid-liquid microextraction combined with high performance liquid chromatography for determination of tetracycline drugs in eggs, Anal. Methods 6 (2014) 6459-6466.

[146] A. Gjelstad, S. Pedersen-Bjergaard, Perspective: hollow fibre liquid-phase microextraction - principles, performance, applicability, and future directions, Sci. Chromatogr. 5 (2013) 181-189.

[147] S.P.M. Ventura, F.A. Silva, M.V. Quental, D. Mondal, M.G. Freire, J.A.P. Coutinho, Ionic-liquid-mediated extraction and separation processes for bioactive compounds: past, present, and future trends, Chem. Rev. 117 (2017) 6984-7052.

[148] R.I. Canales, J.F. Brennecke, Comparison of ionic liquids to conventional organic solvents for extraction of aromatics from aliphatics, J. Chem. Eng. Data 61 (2016) 1685-1699.

[149] G.T. Peres, S. Rath, F.G.R. Reyes, A HPLC with fluorescence detection method for the determination of tetracyclines residues and evaluation of their stability in honey, Food Control 21 (2010) 620-625.

[150] E. Cristofani, C. Antonini, G. Tovo, L. Fioroni, A. Piersanti, R. Galarini, A confirmatory method for the determination of tetracyclines in muscle using highperformance liquid chromatography with diode-array detection, Anal. Chim. Acta 637 (2009) 40-46.

[151] M.J. Schneider, A.M. Darwish, D.W. Freeman, Simultaneous multiresidue determination of tetracyclines and fluoroquinolones in catfish muscle using high performance liquid chromatography with fluorescence detection, Anal. Chim. Acta 586 (2007) 269-274.

[152] W.H. Tsai, T.C. Huang, H.H. Chen, J.J. Huang, M.H. Hsue, H.Y. Chuang, Y.W. Wu, Determination of tetracyclines in surface water and milk by the magnesium hydroxide coprecipitation method, J. Chromatogr. A 1217 (2010) 415-418.

[153] S.P. Khong, Y.A. Hammel, P.A. Guy, Analysis of tetracyclines in honey by highperformance liquid chromatography/tandem mass spectrometry, Rapid Commun. Mass Spectrom. 19 (2005) 493-502.

[154] A. Pena, C.M. Lino, R. Alonso, D. Barceló, Determination of tetracycline antibiotic residues in edible swine tissues by liquid chromatography with spectrofluorometric detection and confirmation by mass spectrometry, J. Agric. Food Chem. 55 (2007) 4973-4979.

[155] Y. Liu, J.Z. Xu, T. Ding, G.H. Li, Determination of tetracyclines in honey using liquid chromatography with ultraviolet absorbance detection and residue confirmation by mass spectrometry, Chin. J. Chem. 25 (2007) 1294-1299.

[156] W.A. Moats, Determination of tetracycline antibiotics in beef and pork tissues using ion-paired liquid chromatography, J. Agric. Food Chem. 48 (2000) 2244-2248.

[157] J. Li, L. Chen, X. Wang, H. Jin, L. Ding, K. Zhang, H. Zhang, Determination of tetracyclines residues in honey by on-line solid-phase extraction high-performance liquid chromatography, Talanta 75 (2008) 1245-1252.

[158] C. Blasco, A.D. Corcia, Y. Picó, Determination of tetracyclines in multi-specie animal tissues by pressurized liquid extraction and liquid chromatography-tandem mass spectrometry, Food Chem. 116 (2009) 1005-1012.

[159] H. Yu, Y. Tao, D. Chen, Y. Wang, Z. Yuan, Development of an HPLC-UV method for the simultaneous determination of tetracyclines in muscle and liver of porcine, chicken and bovine with accelerated solvent extraction, Food Chem. 124 (2011) 1131-1138.

[160] G.H. Wan, H. Cui, H.S. Zheng, J. Zhou, L.J. Liu, X.F. Yu, Determination of tetracyclines residues in honey using high-performance liquid chromatography with potassium permanganate-sodium sulfite- $\beta$-cyclodextrin chemiluminescence detection, J. Chromatogr. B 824 (2005) 57-64.

[161] V.F. Samanidou, K.I. Nikolaidou, I.N. Papadoyannis, Development and validation of an HPLC confirmatory method for the determination of tetracycline antibiotics residues in bovine muscle according to the European Union regulation 2002/657/ EC, J. Sep. Sci. 28 (2005) 2247-2258.

[162] M.I. Lopez, J.S. Pettis, I.B. Smith, P.S. Chu, Multiclass determination and confirmation of antibiotic residues in honey using LC-MS/MS, J. Agric. Food Chem. 56 (2008) 1553-1559.

[163] A. Carrasco-Pancorbo, S. Casado-Terrones, A. Segura-Carretero, A. FernándezGutiérrez, Reversed-phase high-performance liquid chromatography coupled to ultraviolet and electrospray time-of-flight mass spectrometry on-line detection for the separation of eight tetracyclines in honey samples, J. Chromatogr. A 1195 (2008) 107-116.

[164] M.E. Dasenaki, N.S. Thomaidis, Multi-residue determination of seventeen sulfonamides and five tetracyclines in fish tissue using a multi-stage LC-ESI-MS/MS approach based on advanced mass spectrometric techniques, Anal. Chim. Acta 672 (2010) 93-102.

[165] M.J. Schneider, S.E. Braden, I. Reyes-Herrera, D.J. Donoghue, Simultaneous determination of fluoroquinolones and tetracyclines in chicken muscle using HPLC with fluorescence detection, J. Chromatogr. B 846 (2007) 8-13.

[166] A. Pena, N. Pelantova, C.M. Lino, M.I.N. Silveira, P. Solich, Validation of an analytical methodology for determination of oxytetracycline and tetracycline residues in honey by HPLC with fluorescence detection, J. Agric. Food Chem. 53 (2005) 3784-3788.

[167] J.R. Walsh, L.V. Walker, J.J. Webber, Determination of tetracyclines in bovine and porcine muscle by high-performance liquid chromatography using solid-phase extraction, J. Chromatogr. A 596 (1992) 211-216.

[168] M. Gbylik, A. Posyniak, K. Mitrowska, T. Bladek, J. Zmudzki, Multi-residue determination of antibiotics in fish by liquid chromatography-tandem mass spectrometry, Food Addit. Contam. Part A 30 (2013) 940-948.

[169] V.G. Amelin, A.I. Korotkov, High-performance liquid chromatography-high-resolution time-of-flight mass spectrometry in the identification and determination of penicillin and tetracycline group antibiotics in food, J. Anal. Chem. 70 (2015) 1383-1389.

[170] A.R. Shalaby, N.A. Salama, S.H. Abou-Raya, W.H. Emam, F.M. Mehaya, Validation of HPLC method for determination of tetracycline residues in chicken meat and liver, Food Chem. 124 (2011) 1660-1666.

[171] N.V. Eeckhout, J.C. Perez, J. Claereboudt, R. Vandeputte, C.V. Peteghem, Determination of tetracyclines in bovine kidney by liquid chromatography/ tandem mass spectrometry with on-line extraction and clean-up, Rapid Commun. Mass Spectrom. 14 (2000) 280-285.

[172] C. Cháfer-Pericás, A. Maquieira, R. Puchades, B. Company, J. Miralles, A. Moreno Multiresidue determination of antibiotics in aquaculture fish samples by HPLC-MS/MS, Aquacult. Res. 41 (2010) e217-e225.

[173] T. Goto, Y. Ito, S. Yamada, H. Matsumoto, H. Oka, High-throughput analysis of tetracycline and penicillin antibiotics in animal tissues using electrospray tandem ass spectrometry with selected reaction monitoring transition, J. Chromatogr. A 1100 (2005) 193-199.

[174] M. Cherlet, M. Schelkens, S. Croubels, P. De-Backer, Quantitative multi-residue analysis of tetracyclines and their 4-epimers in pig tissues by high-performance liquid chromatography combined with positive-ion electrospray ionization mass spectrometry, Anal. Chim. Acta 492 (2003) 199-213.

[175] P.A. Martos, F. Jayasundara, J. Dolbeer, W. Jin, L. Spilsbury, M. Mitchell, B. Shurmer, Multiclass, multiresidue drug analysis, including aminoglycosides, in animal tissue using liquid chromatography coupled to tandem mass spectrometry, J. Agric. Food Chem. 58 (2010) 5932-5944.

[176] G. Fedorova, V. Nebesky, T. Randak, R. Grabic, Simultaneous determination of 32 antibiotics in aquaculture products using LC-MS/MS, Chem. Pap. 68 (2014) 29-36.

[177] P. Kowalski, Capillary electrophoretic method for the simultaneous determination of tetracycline residues in fish samples, J. Pharm. Biomed. Anal. 47 (2008) 487-493.

[178] P. Masawat, J.M. Slater, The determination of tetracycline residues in food using disposable screen-printed gold electrode (SPGE), Sens. Actuators B 124 (2007) 127-132.

[179] G. Shen, Y. Guo, X. Sun, X. Wang, Electrochemical aptasensor based on prussian blue-chitosan-glutaraldehyde for the sensitive determination of tetracycline, Nano-Micro Lett. 6 (2014) 143-152.

[180] T. Pellinen, G. Bylund, M. Virta, A. Niemi, M. Karp, Detection of traces of tetracyclines from fish with a bioluminescent sensor strain incorporating bacterial luciferase reporter genes, J. Agric. Food Chem. 50 (2002) 4812-4815.

[181] Z. Guo, P. Gai, Development of an ultrasensitive electrochemiluminescence in hibition method for the determination of tetracyclines, Anal. Chim. Acta 688 (2011) 197-202.

[182] S.A. Halvatzis, M.M. Timotheou-Potamia, A.C. Calokerinos, Continuous-flow chemiluminometric determination of tetracyclines in pharmaceutical preparations and honey by oxidation with N-bromosuccinimide, Analyst 118 (1993) 633-637.

[183] N. Pastor-Navarro, S. Morais, A. Maquieira, R. Puchades, Synthesis of haptens and development of a sensitive immunoassay for tetracycline residues: application to honey samples, Anal. Chim. Acta 594 (2007) 211-218.

[184] Y. Shahbazi, F. Ahmadi, N. Karami, Screening, determination and confirmation of tetracycline residues in chicken tissues using four-plate test, ELISA and HPLC-UV methods: comparison between correlation results, Food Agric. Immunol. 26 (2015) 821-834.

[185] M. Jeon, I.R. Paeng, Quantitative detection of tetracycline residues in honey by a simple sensitive immunoassay, Anal. Chim. Acta 626 (2008) 180-185.

[186] R. Mahmoudi, M. Moosavy, R. Norian, S. Kazemi, M.R.A. Nadari, K. Mardani, Detection of oxytetracycline residues in honey samples using ELISA and HPLC methods, J. Pharm. Sci. 19 (2014) 145-150.

[187] P. Masawat, S. Liawruangrath, S. Upalee, Spectrophotometric flow-injection anaysis assay of tetracycline antibiotics using a dual light-emitting diode based detector, Maejo Int. J. Sci. Technol. 2 (2008) 201-209.

[188] P. Zhang, L. Hu, R. Lu, W. Zhou, H. Gao, Application of ionic liquids for liquid-liquid microextraction, Anal. Methods 5 (2013) 5376-5385. 